\title{
Engineering Brain Parasites for Intracellular Delivery of Therapeutic Proteins
}

\author{
Shahar Bracha ${ }^{1, *}$, Karoliina Hassi ${ }^{2}$, Paul D. Ross ${ }^{3,4},{\text { Stuart } \text { Cobb }^{3,4}, \text { Lilach Sheiner }}^{2, *}, \dagger$, Oded \\ $\operatorname{Rechavi}^{1, *, \dagger}$
}

${ }^{1}$ Department of Neurobiology, Wise Faculty of Life Sciences \& Sagol School for Neuroscience, Tel Aviv University, Tel Aviv, Israel.

${ }^{2}$ Wellcome Centre For Molecular Parasitology, Institute of Infection, Immunity \& Inflammation, College of Medical, Veterinary \& Life Sciences, University of Glasgow, UK.

${ }^{3}$ Institute of Neuroscience and Psychology, College of Medical, Veterinary \& Life Sciences, University of Glasgow, Glasgow, UK.

${ }^{4}$ Centre for Discovery Brain Sciences, University of Edinburgh, Edinburgh, UK.

* Correspondence: Shaharbra@gmail.com; Lilach.Sheiner@glasgow.ac.uk; Odedrechavi@gmail.com.

$\uparrow$ Equal contribution.

15 Abstract: Protein therapy has the potential to alleviate many neurological diseases; however, delivery mechanisms for the central nervous system (CNS) are limited, and intracellular delivery poses additional hurdles. To address these challenges, we harnessed the protist parasite Toxoplasma gondii, which can migrate into the CNS and secrete proteins into cells. Using a fusion protein approach, we engineered $T$. gondii to secrete therapeutic proteins for human

20 neurological disorders. We tested two secretion systems, generated fusion proteins that localized to $T$. gondii's secretory organelles and assessed their intracellular targeting in various mammalian cells including neurons. We show that $T$. gondii expressing GRA16 fused to the Rett syndrome protein $\mathrm{MeCP} 2$ deliver a fusion protein that mimics the endogenous $\mathrm{MeCP} 2$, binding heterochromatic DNA in neurons. This demonstrates the potential of $T$. gondii as a therapeutic 25 protein vector, which could provide either transient or chronic, in situ synthesis and delivery of intracellular proteins to the CNS. 


\section{Introduction}

Protein therapeutics have the potential to treat various human disorders: many genetic diseases caused by mutations in a specific protein can be rescued by delivery of the functional protein, treating the root of the disease. In addition, proteins with downstream or compensatory activity can provide indirect rescue or mitigation of disease symptoms (Leader, Baca, \& Golan, 2008). However, owing to their macromolecular nature, the delivery of therapeutic protein to target tissues is extremely challenging. Low functional stability and rapid loss of activity following administration or during storage, along with low permeability through physiological barriers, limit the delivery of active proteins (Leader et al., 2008). Efficient delivery is particularly challenging for neurological diseases due to the blood-brain barrier (BBB), which tightly regulates the transport of molecules to the brain, and blocks the transport of most large, charged and hydrophilic molecules (DiNunzio \& Williams, 2008). In addition to specific delivery to the brain, intracellular delivery of proteins poses additional hurdles. Passive delivery is often precluded as most proteins are unable to spontaneously penetrate through the cell membrane or become endocytosed effectively (Torchilin, 2008). Furthermore, intracellular delivery has been proven especially difficult for neuronal cells (Karra \& Dahm, 2010).

The use of parasites that co-exist with humans for the treatment of chronic and degenerative diseases has a long history. However, these treatments mostly rely on harnessing the ability of the parasite to suppress or induce specific immune responses; examples include the use of immunosuppressive helminths (parasitic worms) for treating allergies and autoimmune diseases (Helmby, 2015); the pre-antibiotics fever therapy for neurosyphilis (Whitrow, 1990) and the use of Bacillus Calmette-Guerin bacteria for cross-vaccination and more recently cancer

50 immunotherapy (Fuge, Vasdev, Allchorne, \& Green, 2015). In addition, the rise of synthetic 
biology offers new methods for bioengineering organisms that mediate designed therapeutic intervention. Some notable examples include metabolically engineered microbiome bacteria (Lu, Mimee, Citorik, \& Pepper, 2017); antigen-presenting microorganisms that induce immune responses against targeted pathogens or tumor cells (Wood \& Paterson, 2014); and the use of engineered viruses for gene therapy (Colella, Ronzitti, \& Mingozzi, 2018).

Toxoplasma gondii is a highly prevalent eukaryotic parasite of the phylum Apicomplexa. Through co-evolution with its hosts, $T$. gondii acquired sophisticated mechanisms to migrate into the CNS and establish quiescent cysts that can persist for the lifetime of the host (Hill, Chirukandoth, \& Dubey, 2005; Mendez \& Koshy, 2017). The primary hosts of T. gondii are felids, but it can infect all warm-blooded organisms as intermediate hosts. In immunocompetent humans, infections are typically asymptomatic or with only mild, short lived flu-like symptoms. As a result, most infections go unnoticed, and it is estimated that about a third of the human population is chronically infected with T. gondii (Pittman \& Knoll, 2015). T. gondii's most common route of entry is by ingestion of infective cysts. Upon entry into the human body, it actively migrates to the brain and passes the BBB by three putative mechanisms: infecting and hitch-hiking on immune cells that infiltrate the CNS through a "trojan horse" mechanism, migrating through the tight junctions of the $\mathrm{BBB}$, or invading into the endothelial cells of the BBB and egressing into the basolateral side (Mendez \& Koshy, 2017). In the brain, T. gondii interacts and resides mostly in neurons (Cabral et al., 2016). Importantly, to survive inside the host, T. gondii secretes a myriad of effector proteins into the host cells and into the extracellular environment (Hakimi, Olias, \& Sibley, 2017).

These characteristics led us to propose the use of $T$. gondii as a biological vector for the delivery of therapeutic proteins to the CNS. T. gondii's active migration can provide high 
specificity to the CNS, and its specialized secretion systems can be used for extracellular or

transgenic $T$. gondii lines that synthesize and deliver therapeutic proteins into mammalian cells. Our work demonstrates that engineered $T$. gondii is a promising biological vector for mediating protein therapy for neurological diseases.

\section{Results}

\section{General approach for engineering $T$. gondii to secrete heterologous proteins}

Our approach for engineering $T$. gondii to secrete heterologous proteins was based on fusing therapeutic proteins to endogenously-secreted proteins of the parasite (Fig. 1). We exploited two $T$. gondii secretion systems that secrete proteins into cells: rhoptries, which inject proteins directly into the cytosol of cells before and during cell invasion (Boothroyd \& Dubremetz, 2008); and dense granules, which secrete proteins after the parasite invaded and resides inside the intracellular parasitophorous vacuole (PV). Dense granule proteins can be secreted continuously throughout $T$. gondii's persistence inside the cell. However, in order for such proteins to reach the host cell from within the PV, they must subsequently be exported through the PV membrane (PVM) and released into the host cell cytosol (Hakimi et al., 2017).

To test these two secretion systems, we selected a series of human and mouse proteins which (a) have validated therapeutic effects in models of human neurological diseases, (b) are required in neurons, (c) are required in relatively low levels, and preferentially (d) have robust in vitro activity assays compatible with $T$. gondii culturing. We also aimed to select proteins of various sizes, target cellular localizations and cellular functions; this allowed us to assess the effect of these protein features on targeting in the parasite, ability to be secreted by the rhoptry or 
dense granules, and export from the PV. According to these criteria, we selected the following proteins: Aspartoacylase $(A S P A, 36 \mathrm{kDa})$, a nuclear-cytosolic enzyme associated with Canavan disease (Leone et al., 2012); Survival of Motor Neuron (SMN1, 32kDa), a nuclear-cytosolic 100 protein associated with spinal muscular atrophy (Bowerman et al., 2017); Glial Derived Neurotrophic factor $(G D N F, 24 \mathrm{kDa})$, a signaling peptide associated with neuroregeneration and a variety of neurodegenerative diseases (Allen, Watson, Shoemark, Barua, \& Patel, 2013); Parkin (PARK2, 42kDa), a nuclear-cytosolic E3 ubiquitin-ligase associated with Parkinson's disease (Mochizuki, 2007); Galactosylceramidase (GALC, 77kDa), a lysosomal enzyme associated with Krabbe disease (Rafi, Rao, Luzi, Curtis, \& Wenger, 2012), Transcription factor $\mathrm{EB}(T F E B, 53 \mathrm{kDa})$, a nuclear-cytosolic transcription factor associated with a variety of neurodegenerative and lysosomal storage diseases (Napolitano \& Ballabio, 2016); and MethylCpG binding Protein $2(M E C P 2,54 \mathrm{kDa})$, a nuclear DNA-binding protein associated with Rett syndrome (Katz et al., 2016). To examine the effect of codon usage on expression levels and targeting of the heterologous proteins, the mammalian genes $A S P A, G A L C$ and $M E C P 2$ were tested in both their native mammalian sequence and codon optimized according to $T$. gondii's codon usage (labeled 'opt', e.g. MECP2opt). The lysosomal GALC was tested in an additional variation containing a TAT protein transduction domain, which promotes protein transduction across membranes and improves cross-correction between cells (Meng, Eto, Schiffmann, \& Shen, 2013). 


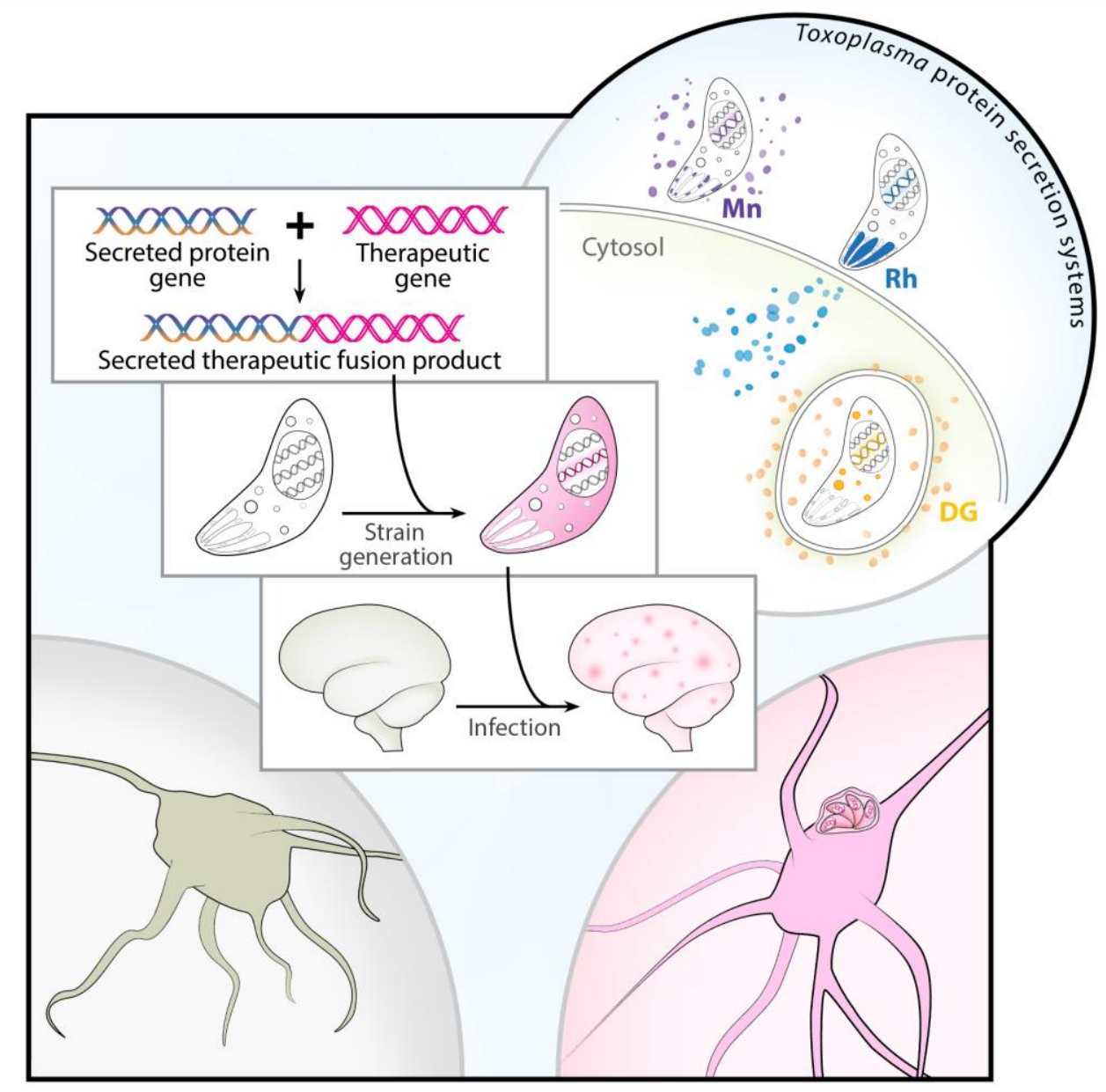

Fig. 1. Generating T. gondii for delivery of heterologous therapeutic proteins.

A therapeutic protein of interest is genetically fused to an endogenously-secreted protein of the

construct encoding for the fusion protein is introduced into $T$. gondii to generate transgenic lines which secrete the therapeutic protein. The transgenic $T$. gondii actively migrates to the CNS, bypasses the BBB and delivers the protein to neurons. The top right corner presents the three secretion systems of $T$. gondii, which secrete proteins using different mechanisms and at different stages of cell invasion or intracellular persistence. Mn- microneme, Rh- rhoptry, DGdense granule. 


\section{Targeting proteins to the rhoptry secretory organelle for transient delivery}

The first secretion system we tested was the rhoptries. As some rhoptry proteins are injected before cell invasion, their secretion does not depend on productive invasion and persistence of the parasite inside the host cell. Furthermore, quantification in the CNS of mice showed that rhoptry proteins can be injected by $T$. gondii into 30-50 times more cells in the brain than those productively infected (Koshy et al., 2012). This suggests an advantage of rhoptry secretion for transient delivery of proteins, as each parasite can secrete rhoptry proteins into multiple cells without invading them.

In order to co-opt rhoptry secretion, heterologous proteins were fused to the rhoptry protein Toxofilin, which is injected into the cytosol of host cells upon initial parasite engagement (Boothroyd \& Dubremetz, 2008). The fusion to Toxofillin was modelled after an ingenious reporter line of $T$. gondii expressing Toxofilin-fused Cre recombinase, which enabled the

140 labeling of cells contacted by the parasite both in vitro and in vivo (Cabral et al., 2016; Koshy et al., 2010; Lodoen, Gerke, \& Boothroyd, 2010). We generated parasites with genomic integration of constructs encoding for Toxofilin fused to different variations of: ASPA, SMN, GDNF, PARK2, GALC, MECP2, and TFEB (Table S1). Expression was controlled by the endogenous promoter of Toxofilin, and an HA tag was added for immunolabeling of the fusion proteins. Most

145 fusion proteins mis-localized to other organelles of the parasite, predominantly to the endoplasmic reticulum (ER), golgi and micronemes. However, Toxofilin-fused GDNF, PARK2 and codon-optimized TFEB (TFEBopt) were successfully localized to the rhoptries (Fig. 2,

\section{Table S1).}


A

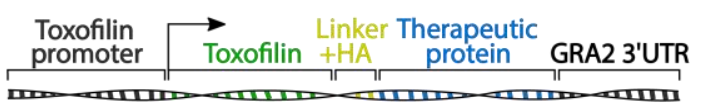

C

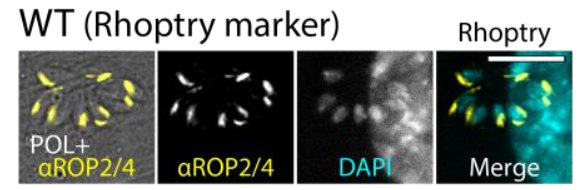

D Toxofilin-HA-ASPA

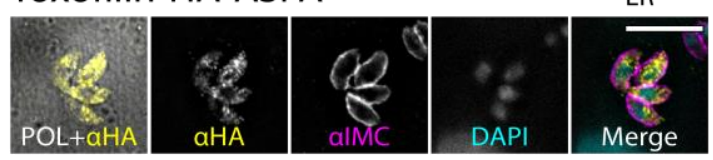

Toxofilin-HA-ASPAopt

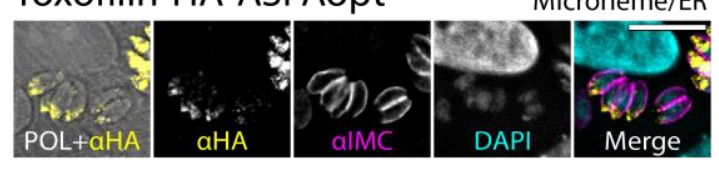

Toxofilin-HA-GALC

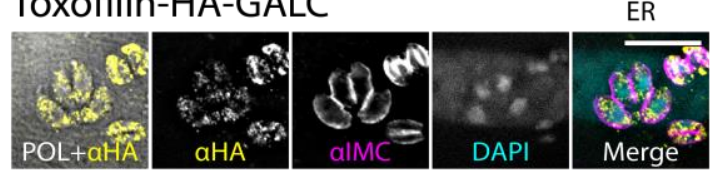

Toxofilin-HA-GALCopt

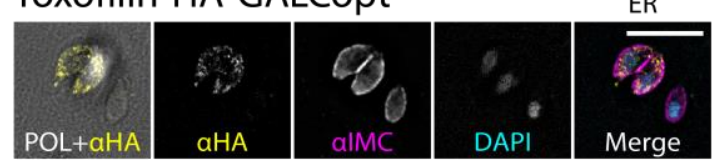

Toxofilin-HA-GALC-TAT

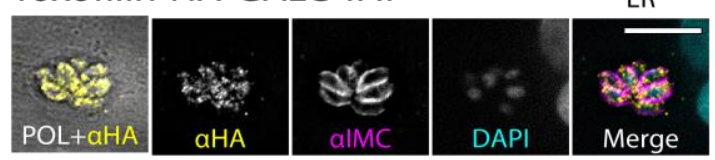

Toxofilin-HA-SMN1

150

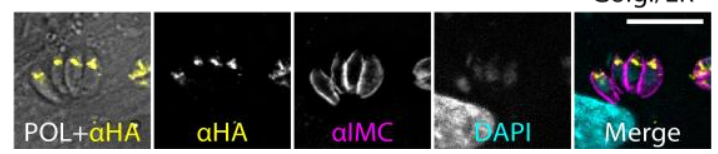

B
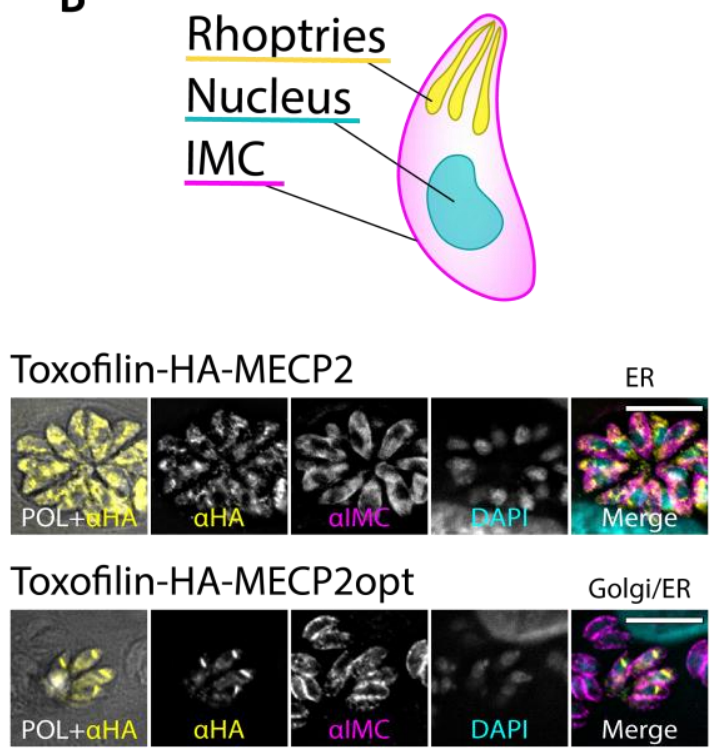

Toxofilin-HA-GDNF

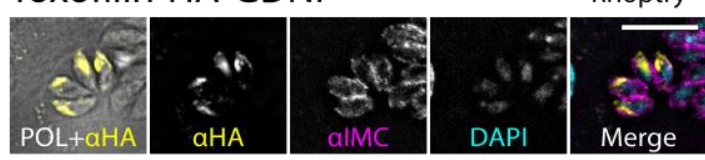

Toxofilin-HA-PARK2

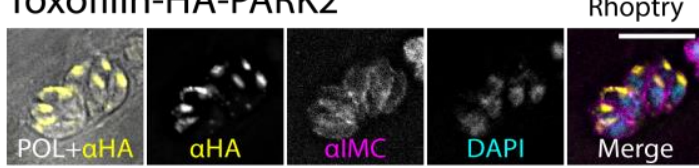

Toxofilin-HA-TFEBopt

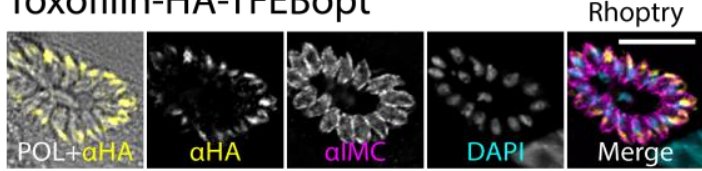

Fig. 2. Targeting of therapeutic proteins to $T$. gondii's rhoptries by fusion to Toxofilin.

(A) Scheme of the genetic construct used to generate $T$. gondii expressing Toxofilin-fused

therapeutic proteins. (B) Illustration of a $T$. gondii parasite. IMC- inner membrane complex,

marking the parasite outline. (C) Intracellular T. gondii immunostained with the rhoptry marker

155 anti-ROP2/4. (D) Intracellular $T$. gondii stably expressing different Toxofilin-fused proteins

related to human neurological diseases. Selected images represent localization of the proteins to 
the rhoptries (TFEBopt, PARK2, GDNF) or in the lack of any, the most common localizations in the stable pool (full list of observed localizations in Table S1). Text above the merge images indicates the localization of the fusion protein in the image. Scale bar: $10 \mu \mathrm{m}$.

\section{Targeting proteins to the dense granule secretory organelle for continuous delivery}

As rhoptry secretion occurs as a discrete event upon initial parasite engagement, it provides only transient protein delivery. In contrast, dense granule proteins can be secreted continuously as the parasite persists inside a cell. Therefore, we assessed dense granule-mediated secretion for continuous delivery of intracellular proteins. For this purpose, we designed translational fusions to GRA16. GRA16 is secreted from the dense granules to the parasitophorous vacuoles (PV), exported from the PV to the host cell cytosol, and localizes to the host cell nucleus (Bougdour et al., 2013). We generated parasites with genomic integration of constructs encoding for GRA16 fused to different variants of: ASPA, SMN, GALC, MECP2, and TFEB (Table S2). Expression was controlled by the endogenous promoter of GRA16, and an HA tag was added for immunolabeling of the fusion proteins.

GRA16-fused GALC-TAT was not expressed in T. gondii. GRA16-fused GALCopt was expressed, but localized only to the ER of the parasites and was not secreted to the PV, nor detected in the host cell. ASPA, ASPAopt and GALC fused to GRA16 localized to the PV but were not detected in the host cell, indicating that they were successfully targeted to the dense granules and secreted to the PV, but not exported in detectable levels. Importantly, the GRA16fused nuclear proteins MeCP2 (MECP2opt, total fusion protein size 110kDa), SMN (SMN1, $88 \mathrm{kDa})$ and TFEB (TFEBopt, 109kDa) localized to both the PV and the host cell nucleus. This indicates that these proteins were successfully targeted to the dense granules, secreted to the PV, 
180 exported to the host cell cytosol and accumulated in their site of activity, the host nucleus (Fig.

3) (Bowerman et al., 2017; Katz et al., 2016; Napolitano \& Ballabio, 2016).

Interestingly, by analyzing the level of intrinsic disorder for each of the tested fusion proteins, we found a strong correlation $\left(\mathrm{R}=0.975, \mathrm{P}=4 * 10^{-5}\right)$ between the level of localization to the host cell nucleus and the average intrinsic disorder score of the fused protein (based on

185 IUPred2A (Mészáros, Erdős, \& Dosztányi, 2018)) (Fig. 3F, Fig. S1). This provides strong support for involvement of protein structural disorder in export through the PVM, and for the hypothesized translocon model of protein export (Hakimi \& Bougdour, 2015; Hakimi et al., 2017).

To assess whether a shorter fragment of GRA16 could replace the full-length protein as a 190 carrier for secretion, we tested three truncated variants of GRA16 fused to MeCP2. However, as none of the truncated fusion proteins were detected in the host cell nucleus, we decided to continue with the full-length GRA16 as the carrier protein (Fig. S2).

Since GRA16-fused MeCP2 and TFEB displayed the most robust delivery and targeting to the host cell nucleus, we focused on these fusion proteins for the rest of the study. The $T$.

195 gondii lines expressing these proteins were named GRA16-MeCP2 (expressing GRA16-HAMECP2opt) and GRA16-TFEB (expressing GRA16-HA-TFEBopt). In addition, T. gondii expressing HA-tagged GRA16 which is not fused to any protein (GRA16-HA) was generated as a control. 


\section{A \\ C \\ D}
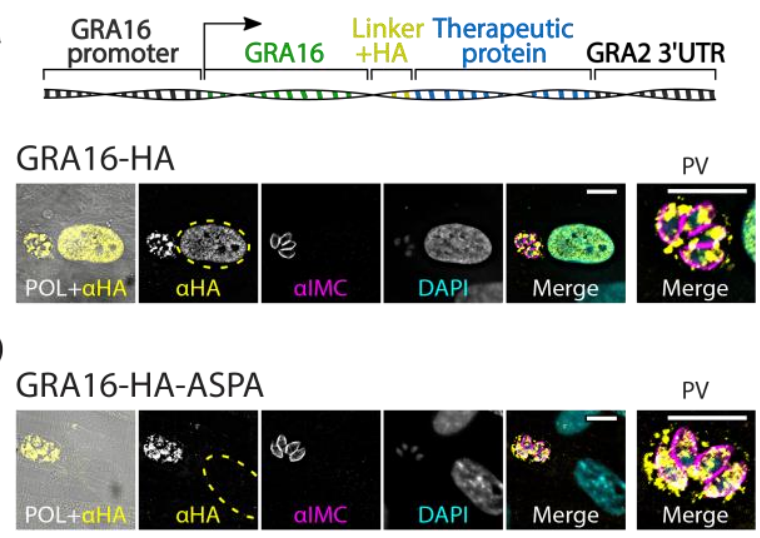

GRA16-HA-ASPAopt
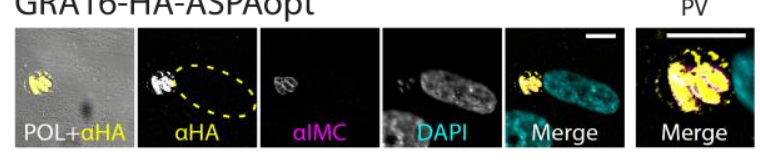

GRA16-HA-GALC
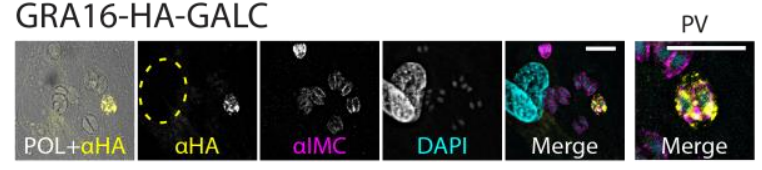

GRA16-HA-GALCopt
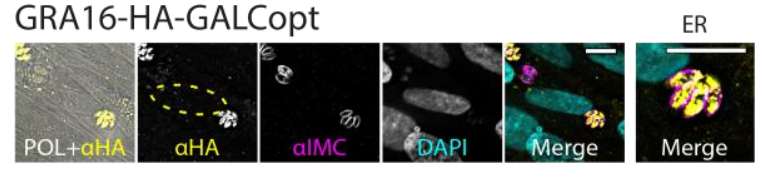

E

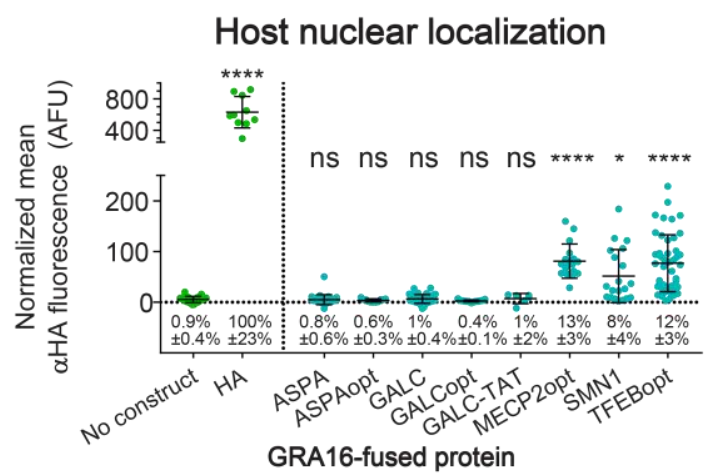

B

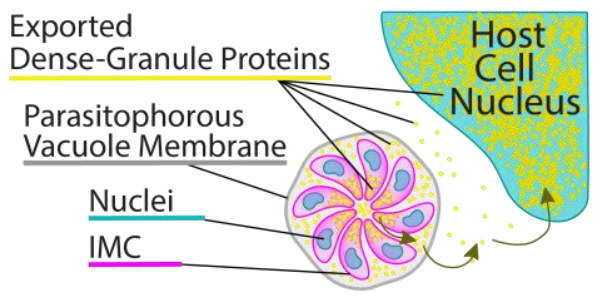

GRA16-HA-GALC-TAT

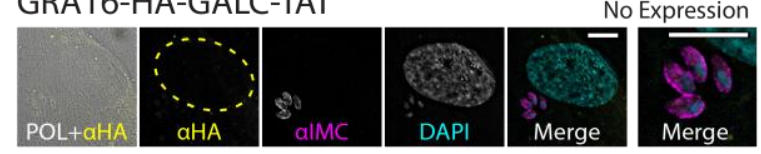

GRA16-HA-MECP2opt

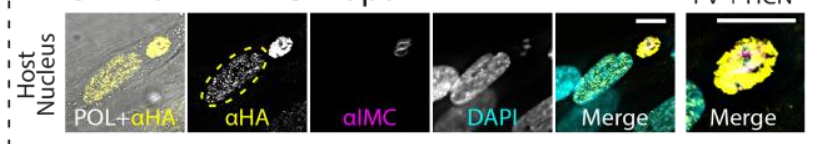

\section{GRA16-HA-SMN1}
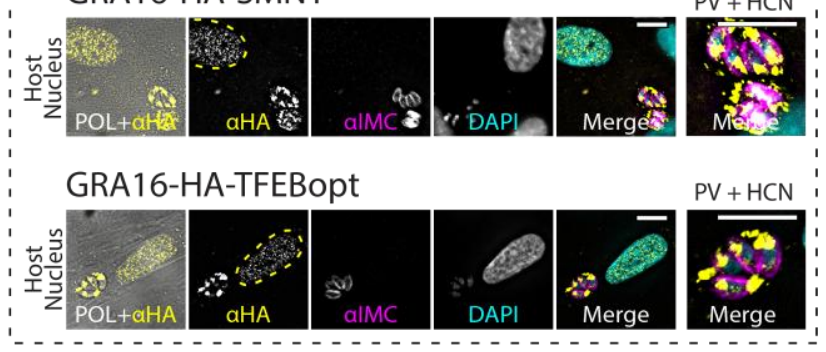

F Protein disorder vs. Host nuclear localization

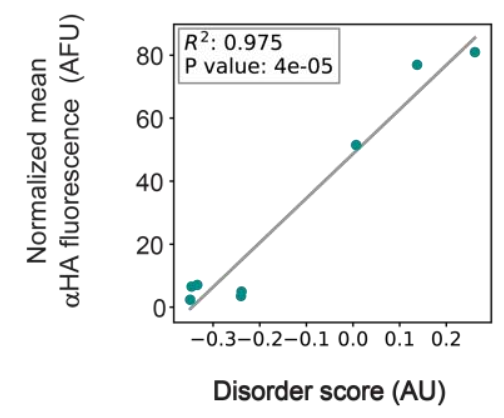

Fig. 3. Targeting of therapeutic proteins to $T$. gondii's dense granules, parasitophorous vacuole and host cell nucleus by fusion to GRA16.

(A) Scheme of the genetic construct used to generate T. gondii expressing GRA16-fused

therapeutic proteins. (B) Illustration of an intracellular parasitophorous vacuole containing eight

T. gondii parasites. Yellow- exported dense-granule protein targeted to the nucleus of the host 
cell. (C-D) Intracellular T. gondii stably expressing HA-tagged GRA16 (C) or different GRA16fused proteins related to human neurological diseases (D). Selected images represent localization of the proteins to the PV and host cell nucleus (TFEBopt, MECP2opt, SMN1), to the PV alone (ASPA, ASPAopt, GALC) or in the lack of any, the most common localizations in the stable pool (full list of observed localizations in Table S2). Rightmost image of each set shows a closeup of the parasite vacuole, and the text above it indicates the localizations of the fusion protein in the image. Yellow dashed lines mark the host cell nucleus $(\mathrm{HCN})$. (E) Fluorescence quantification of the anti-HA signal in the nucleus of infected host cells. Error bars represent mean \pm SD. $N=6-47$ cells per condition. Take note that the $y$-axis is divided to two segments, to account for the high values recorded from GRA16-HA. AFU- arbitrary fluorescence units. Text below each scatter plot shows the mean relative nuclear localization $\pm 95 \%$ confidence interval (relative to GRA16-HA). Significance represents the difference between the fusion protein and the parental strain ('no construct'), calculated by one-way ANOVA with multiple comparisons (Dunnett test). $* * * * \mathrm{P}<0.0001 ; * \mathrm{P}<0.0332$; ns- not significant. (F) Correlation between host nuclear localization and the level of intrinsic disorder of the fused therapeutic protein, based on IUPred2A (see Fig. S1). Linear regression line drawn in grey. Scale bar: $10 \mu \mathrm{m}$.

\section{Kinetics of GRA16-mediated protein delivery in human fibroblasts and neurons}

To characterize the kinetics of GRA16-mediated protein delivery in vitro, we used highcontent imaging to quantify infection rate and nuclear protein delivery for parasites expressing GRA16 alone and GRA16 fused to MeCP2 or TFEB. We infected human foreskin fibroblast (HFF) cells using different MOI (Multiplicity Of Infection- the proportion of parasite per host cell administered to the culture) and followed nuclear fluorescence over time (Fig. 4A-C). 
Overall, there was no significant difference in the infection rate of the different lines, indicating that they are similar in their ability to invade host cells (by two-way ANOVA). 24 hours postadministration, the percentage of cells productively infected was on average $50-65 \%$ for cells infected using $\mathrm{MOI}=3,29-48 \%$ with $\mathrm{MOI}=1$ and 13-19\% with $\mathrm{MOI}=0.33 .24$ hours postadministration, the mean percentage of host cell nuclei labeled as positive for the proteins was $44-53 \%$ for cells infected using a MOI=3, 20-43\% with $\mathrm{MOI}=1$ and 8-21\% with $\mathrm{MOI}=0.33$. Quantification of fluorescence intensity in the host cell nuclei showed that the fusion of the mammalian proteins MeCP2 and TFEB to GRA16 reduced its accumulation in the host cell nucleus by 7.5 folds on average.

\section{Delivery of GRA16-fused TFEB and MeCP2 to the nucleus of human neurons} transgenic $T$. gondii can deliver therapeutic proteins to their site of activity in human neurons. Lund Human Mesencephalic (LUHMES) cells were differentiated in vitro to morphologically and biochemically mature dopamine-like neurons (Lotharius et al., 2002; Scholz et al., 2011; Shah et al., 2016) and infected with the T. gondii lines GRA16-HA, GRA16-MeCP2 and

GRA16-TFEB. All tested lines displayed clear secretion, export and accumulation of the tagged protein in the nuclei of the neurons. Analysis of the kinetics of parasite infection in these neurons showed that 24 hours post-administration, $70 \%$ of neurons were infected using MOI=2, 59\% using $\mathrm{MOI}=1$ and $39 \%$ using $\mathrm{MOI}=0.5$ (Fig. 4D-F). 
A

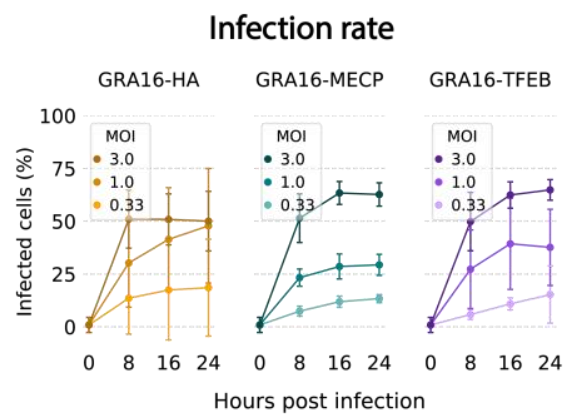

B

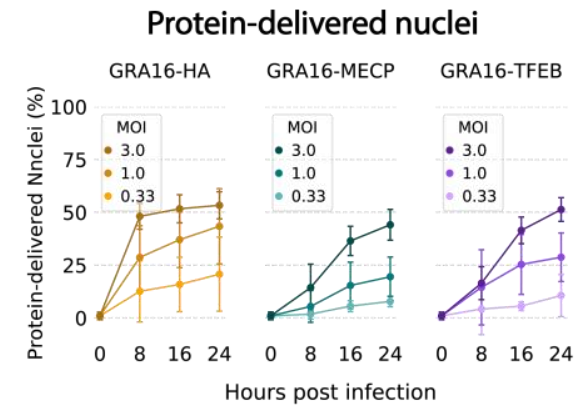

C

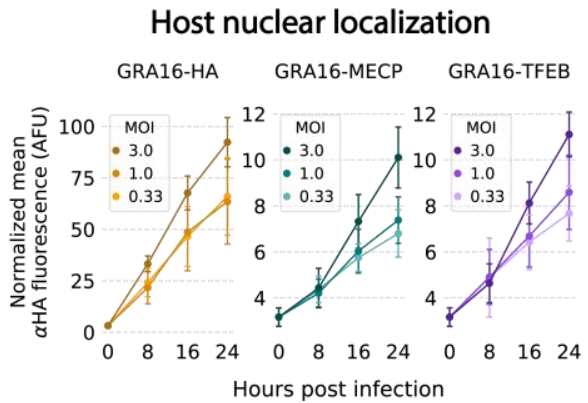

D Human neurons GRA16-HA-MECP2

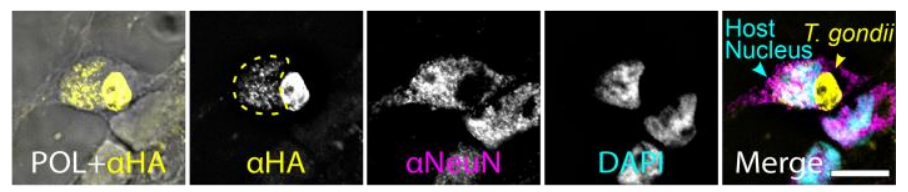

GRA16-HA-TFEB

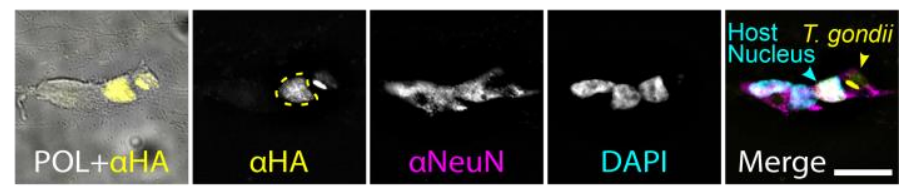

GRA16-HA

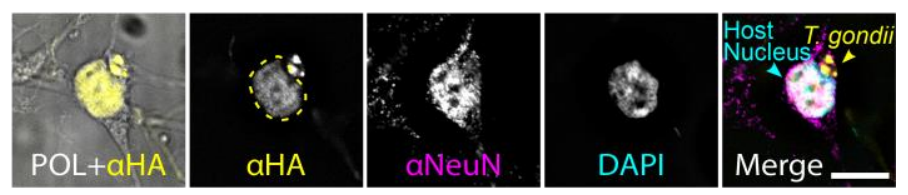

E

$\mathbf{F}$

Infection rate GRA16-HA
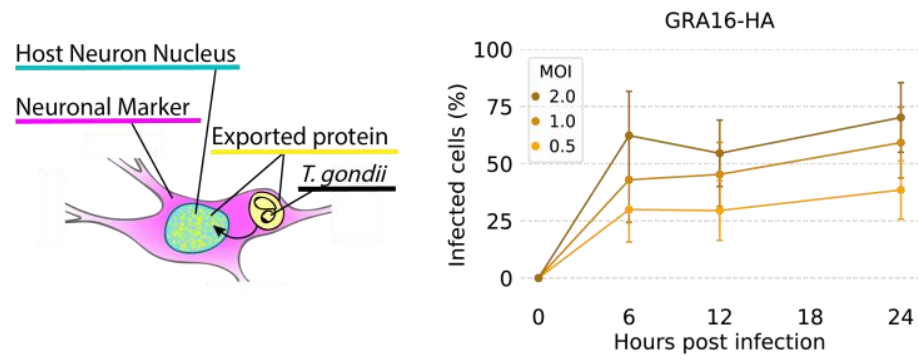

Fig. 4. The kinetics of cell infection and protein delivery in human fibroblasts and neurons.

(A-C) Quantitative characterization of the kinetics of cell infection and protein delivery in human foreskin fibroblasts (HFF), infected with different concentrations (MOI) of $T$. gondii expressing GRA16-HA, GRA16-MeCP2 and GRA16-TFEB. (A) Infection rate over time. (B) Percentage of HFF nuclei labelled positive for the delivered protein over time. (C) Normalized mean fluorescence intensity in the nuclei of host cells infected with a single parasite vacuole, over time. Error bars represent mean $\pm \mathrm{SD}$. N=24-40 wells per condition (average 1800 cells per well). (D) Human neurons differentiated from Lund Human Mesencephalic (LUHMES) cells, infected with T. gondii expressing GRA16-HA, GRA16-MeCP2 and GRA16-TFEB. Yellow 


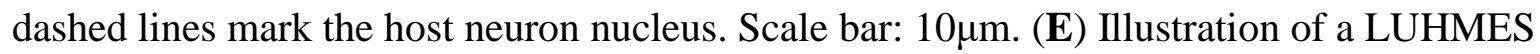

neuron infected with T. gondii. Yellow- exported dense-granule protein targeted to the nucleus of the neuron. (F) Infection rate in LUHMES neurons infected with T. gondii expressing GRA16HA, over time. Error bars represent mean \pm SD. $N=10-60$ images per condition (average 80 cells per image).

\section{T. gondii-delivered MeCP2 binds heterochromatic DNA in the nucleus of primary neurons}

In order to test the therapeutic relevance of the system, we focused on GRA16-MeCP2. $\mathrm{MeCP} 2$ is a nuclear protein which is expressed ubiquitously, but MeCP2 levels are highest in postmitotic neurons, where it is critical for the function and maintenance of the neurons (Lyst \& Bird, 2015). This is demonstrated by the disease manifestation of Rett syndrome (RTT), a severe neurological disorder caused by loss-of-function mutations in the $M E C P 2$ gene. The activity of the MeCP2 protein depends on its specific binding of heterochromatic DNA in the nucleus. $\mathrm{MeCP} 2$ recognizes patterns of methylated DNA and recruits co-repressor complexes. Therefore, a classical readout for its functionality is its ability to specifically bind heterochromatic DNA

275 (Guy et al., 2018).

To test the functionality of GRA16-fused MeCP2, we infected mouse primary neuronal cultures with $T$. gondii expressing GRA16-MeCP2. In mouse cells, heterochromatic DNA can be readily visualized with DAPI staining. Importantly, we found that the MeCP2 fusion protein delivered by the parasites co-localizes to the foci of heterochromatic DNA in the nucleus (Fig. 5A). This suggests that the MeCP2 fusion protein synthesized and delivered by the parasites binds heterochromatic DNA in the neuronal nucleus, mimicking the functional endogenous MeCP2 (Guy et al., 2018). Similarly, mouse neuroblastoma (N2A) cells infected with T. gondii 
secreting GRA16-MeCP2 presented the same binding of heterochromatic DNA, as well as colocalization with MeCP2 immunostaining. By comparison, cells infected with $T$. gondii parasites secreting GRA16-HA did not present the same co-localization, and the protein was evenly diffused in the nucleus, confirming the specificity of binding to the fusion protein containing $\mathrm{MeCP} 2$ (Fig. 5B).

Excess of MeCP2 can be deleterious, as exhibited by the pathogenesis of MECP2 duplication syndrome (Ramocki, Tavyev, \& Peters, 2010). Furthermore, gene therapy studies have shown that MECP2 overexpression leading to 2-6 folds higher protein levels can induce adverse symptoms and increased mortality in mice (Collins et al., 2004; Gadalla et al., 2013; Luikenhuis, Giacometti, Beard, \& Jaenisch, 2004). To test the level of MeCP2 rescue achieved by the transgenic T. gondii, we quantified MeCP2 delivery in MeCP2-deficient neurons. MeCP2 knock-out (MECP2-KO) LUHMES cells (Shah et al., 2016) were differentiated into mature 295 human neurons and infected with GRA16-MeCP2 T. gondii. Nuclear levels of MeCP2 in infected and non-infected neurons were quantified by immunofluorescence, and relative protein levels were calculated by comparison to endogenous MeCP2 in wild-type (WT) neurons. We found that 12 hours post-infection T. gondii-mediated MeCP2 rescue reaches on average $44 \%$ of WT levels, and 24 hours post-infection it reaches $58 \%$ of WT levels $(\mathrm{P}<0.0001$, two-way ANOVA). Importantly, MeCP2-KO neurons infected with GRA16-HA T. gondii do not show a significant increase in anti-MeCP2 immunostaining (12 hours: $\mathrm{P}=0.88,24$ hours: $\mathrm{P}=0.35$ ) (Fig. 5C-D). This suggests that the amount of protein delivered by transgenic T. gondii lies within a range compatible with therapeutic benefit (Carrette, Blum, Ma, Kelleher, \& Lee, 2018; Gadalla et al., 2013). 
A

\section{Mouse primary neurons}

Infected with GRA16-HA-MeCP2

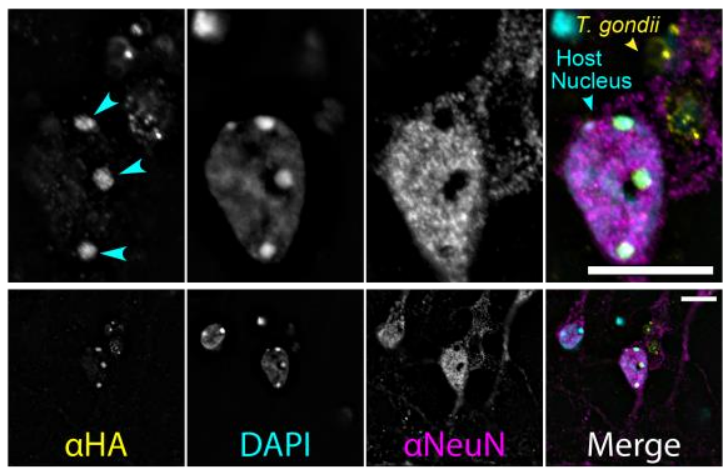

C WT LUHMES human neurons Uninfected

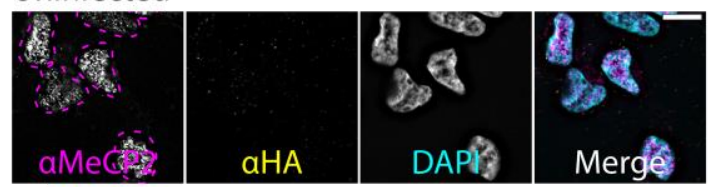

MeCP2-KO LUHMES human neurons Infected with GRA16-HA-MeCP2

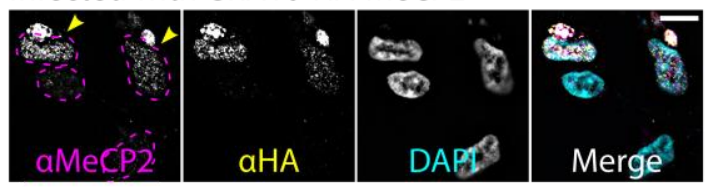

MeCP2-KO LUHMES human neurons

Infected with GRA16-HA (control)

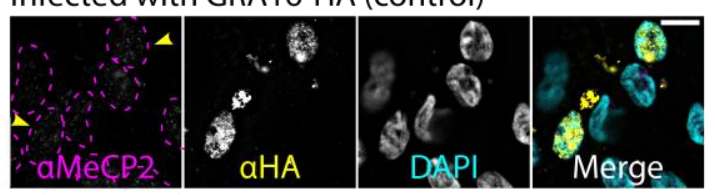

B

Mouse neuroblastoma (N2A) cells

Infected with GRA16-HA-MeCP2

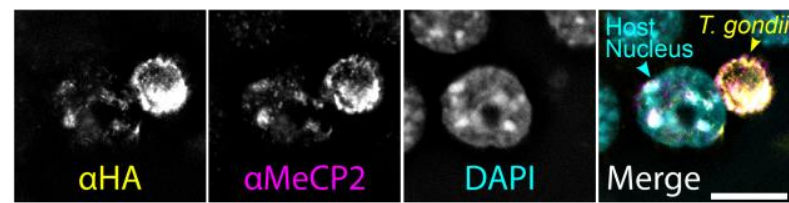

Infected with GRA16-HA (control)

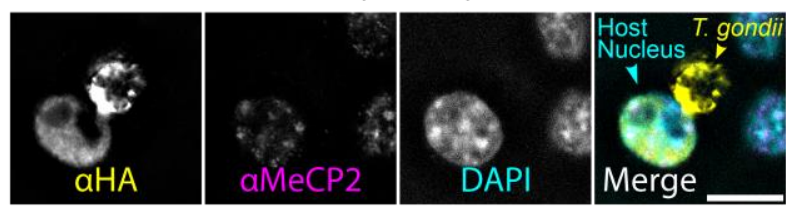

D

Nuclear MeCP2 levels

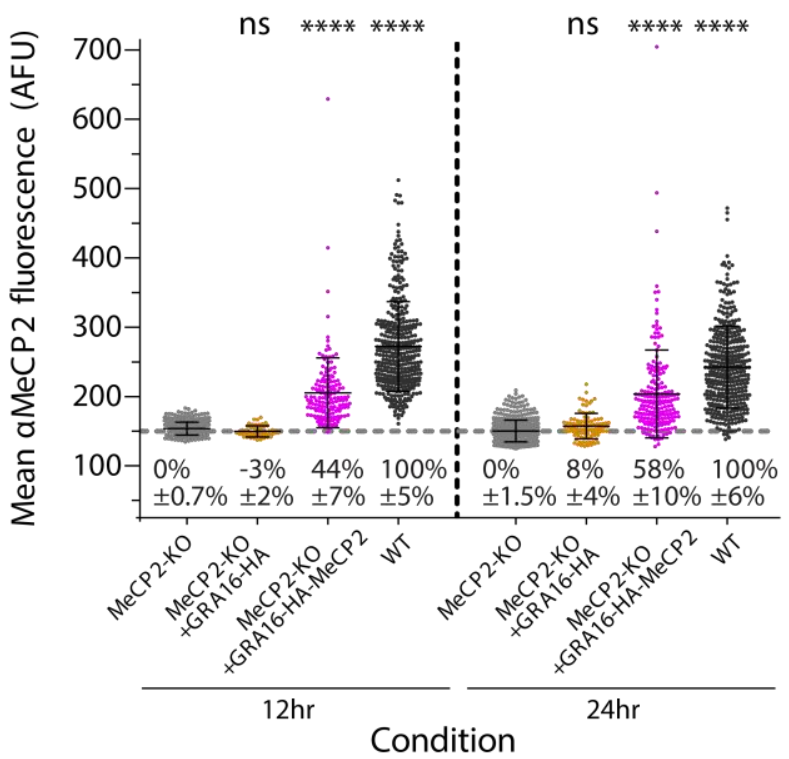

Fig. 5. GRA16-MeCP2 binds heterochromatic DNA in neurons and is delivered in therapeutic levels.

(A) Mouse primary neurons infected with GRA16-MeCP2 T. gondii, 12 hours after inoculation. Top images show a close-up of the neuronal soma and intracellular $T$. gondii. Blue arrows mark co-localization of the GRA16-MeCP2 protein with foci of heterochromatic DNA. (B) N2A mouse neuroblastoma cells infected with GRA16-MeCP2 and GRA16-HA T. gondii, 24 hours after inoculation. (C) LUHMES WT and MeCP2-KO human neurons infected with GRA16- 
MeCP2 and GRA16-HA T. gondii, 12 hours after inoculation. Magenta dashed lines mark the neuronal nuclei. Yellow arrows mark infected neurons. (D) Fluorescent quantification of the anti-MeCP2 signal in the nucleus of MeCP2-KO neurons, MeCP2-KO neurons infected with GRA16-HA (negative control) or GRA16-MeCP2 T. gondii, and WT neurons. Error bars represent mean \pm SD. $\mathrm{N}=50-510$ cells per condition. AFU- arbitrary fluorescence units. Text below each scatter plot shows the mean relative nuclear levels of MeCP2 (compared to WT neurons) $\pm 95 \%$ confidence interval. Significance represents the difference between each condition and the untreated MeCP2-KO neurons, calculated by two-way ANOVA with multiple comparisons (Dunnett test). $* * * * \mathrm{P}<0.0001$; ns- not significant. Scale bar: $10 \mu \mathrm{m}$.

\section{Discussion}

In this study we investigated a method for therapeutic intracellular protein delivery via a transgenic brain parasite $-T$. gondii. We assessed different approaches for heterologous protein secretion and characterized $T$. gondii lines that deliver therapeutic proteins to the nuclei of various mammalian cells. We showed that $T$. gondii expressing a GRA16-fused MeCP2 deliver a fusion protein that binds heterochromatic DNA in neurons, mimicking the functional endogenous $\mathrm{MeCP} 2$.

For both the Toxofilin and GRA16 fusion proteins, we found that mistargeted proteins were mostly arrested within the secretory pathway (ER, golgi). However, some fusion proteins resulted in unexpected mistargeting to parasite-unique organelles, such as the microneme or apicoplast (Table S1 and S2). As the fusion proteins tested in this study represent a wide and unconventional set of sequences, the targeting of the tested fusion proteins could provide an 
interesting resource to analyze factors affecting protein trafficking in $T$. gondii, as well as the regulation of protein secretion and export from the parasitophorous vacuole (PV).

Interestingly, previous work which attempted to fuse dense granule exported proteins to marker proteins such as fluorescent or other highly structured proteins found that fusion to another protein blocked its ability to be exported through the PV membrane (PVM), causing it to accumulate in the PV space (Curt-Varesano, Braun, Ranquet, Hakimi, \& Bougdour, 2016; Hakimi \& Bougdour, 2015; Hakimi et al., 2017; Marino et al., 2018). In addition, known exported dense granule proteins of $T$. gondii are enriched in regions of high intrinsic disorder (Hakimi \& Bougdour, 2015). In this study we tested the secretion, export and host cell nuclear localization of multiple heterologous proteins fused to the dense granule exported protein GRA16. Of the expressed fusion proteins that were successfully secreted by the dense granules, some were detected in the host cell nucleus (MeCP2, SMN, TFEB) and some were not, suggesting that they were not exported from the PV (ASPA and GALC). When comparing the intrinsic disorder profiles of these proteins, we noticed a significant correlation between the level of protein disorder and the propensity to be exported and localize to the host cell nucleus (Fig. 3, Fig. S1). This provides strong support for the proposed model of protein export, in which high intrinsic disorder is required for protein export through the PVM (Hakimi et al., 2017).

During this study we also established a new model for the study of $T$. gondii in human neurons differentiated from neuronal precursor LUHMES cells. Although for many hosts

355 (including humans), T. gondii's dominant infection is in the CNS, most research on the biology of $T$. gondii is performed in standard fibroblast and epithelial cell lines. On the other hand, use of primary neuronal cultures can be highly variable, expensive, labor intensive and lead to high animal use. LUHMES cells are conditionally-immortalized neuronal precursor cells for which 
culturing is straightforward and which can differentiate into mature dopamine-like human neurons within one week (Lotharius et al., 2002; Scholz et al., 2011; Shah et al., 2016). This LUHMES neuronal model could facilitate the study of fundamental questions regarding the unique interaction of $T$. gondii with neurons, and especially human neurons.

T. gondii-mediated protein delivery has multiple attractive characteristics. $T$. gondii has an exceptionally high CNS-specificity driven by active trafficking to the CNS from peripheral tissues, even with oral administration. Likewise, active motility also drives $T$. gondii's widespread distribution in the brain - in a systematic characterization of cyst distribution in infected mice, $92 \%$ of the brain regions examined were found to contain tissue cysts (Berenreiterová, Flegr, Kuběna, \& Němec, 2011). Transgenic T. gondii can provide either longterm or transient protein delivery, based on the utilized secretion organelle and subsequent persistence or clearance of the parasites. Importantly, as a biological agent, $T$. gondii is responsive to external cues and can implement engineered genetic circuits (Jimenez-Ruiz, Wong, Pall, \& Meissner, 2014; Wang et al., 2016). This could be utilized for incorporating inducible expression systems, or for generating attenuated $T$. gondii which can be cleared from the tissue following protein delivery. T. gondii can provide local and compartmentalized protein synthesis in situ and deliver proteins directly into the cytosol of cells. It can deliver large proteins, the size limit of which is yet unknown (the largest fusion protein delivered in this study is 110kDa). For comparison, the leading vectors used for gene therapy today, AAV and self-complementary AAV (scAAV), are limited to a packaging capacity of $4.7 \mathrm{~kb}$ and $2.2-3 \mathrm{~kb}$, respectively, which renders them impractical for many human proteins (Colella et al., 2018). 
apparently normal early development followed by profound neurologic regression around 1-2 years of age (Carrette et al., 2018; Katz et al., 2016). Since 2007, several gene therapy studies in rodents have shown that expression of functional MeCP2 can reverse the symptoms of Rett syndrome, even after disease onset (Gadalla et al., 2013, 2017; Guy, Gan, Selfridge, Cobb, \& Bird, 2007; Katz et al., 2016). Furthermore, exclusive MeCP2 expression in the CNS is sufficient to rescue the majority of Rett syndrome phenotypes (Ross et al., 2016), supporting the therapeutic potential of $\mathrm{MeCP} 2$ delivery to the CNS. As a result, several studies are exploring $M E C P 2$ gene therapy, mostly using AAV and scAAV vectors for delivery of the $M E C P 2$ gene 390 (Gadalla et al., 2013; Garg et al., 2013; Katz et al., 2016; Matagne et al., 2017; Sinnett et al., 2017). These studies have been spurring tremendous hope for gene therapy of Rett syndrome. However, some challenges are still being overcome, such as toxicity from overexpression or mistargeting of the gene, packaging limitations of the viral vectors and limited brain transduction efficiency (Gadalla et al., 2017; Katz et al., 2016). In this study, we demonstrate intracellular delivery of the $\mathrm{MeCP} 2$ protein into neurons. Importantly, we also demonstrate that the delivered $\mathrm{MeCP} 2$ protein binds heterochromatic DNA in the neurons, mimicking the functional endogenous MeCP2 (Carrette et al., 2018; Gadalla et al., 2013).

In this study we developed and characterized transgenic $T$. gondii lines capable of intracellular delivery of therapeutic proteins based on fusion to GRA16. This approach could be extended to the use of other endogenously-secreted $T$. gondii proteins which exhibit different secretion dynamics and could be adapted to the therapeutic protein of interest. Furthermore, in addition to the delivery of endogenous mammalian proteins, this approach could be used for the delivery of engineered proteins, such as engineered enzymes, ligands, antibodies, transcriptional regulators or programmable nucleases (Gaj, Gersbach, \& Barbas, 2013). 


\section{Acknowledgements:}

We thank all members of the Rechavi and Sheiner labs for their endless help, support, enriching discussions and valuable feedback on the manuscript. We thank Prof. Daniel Frenkel and Prof. Dan Peer from Tel Aviv University for their advice. Dror Cohen from Tel Aviv University for

410 the illustration of figure 1. Dr. Eric Kalkman and Susan Baillie from the Scottish Bioscreening Facility for technical help with the automated high-content imaging systems. Prof. Anita Koshy from the University of Arizona for the Toxofilin-Cre plasmid and Dr. Ruth Shah and Prof. Adrian Bird from the University of Edinburgh for the LUHMES cell lines. This work was supported by the Nadal-Colton Applied Research Fund, International Rett Syndrome Foundation

415 (Rettsyndrome.org), Adelis Foundation, Glasgow Knowledge Exchange Fund, Naomi Kadar Foundation Fellowship, Joan and Jaime Constantiner Fellowship and Prajs-Drimmer Scholarship. L.S. is a Royal Society of Edinburgh personal research fellow.

\section{Author Contribution:}

420 S.B., O.R. and L.S. conceived the study and designed the experiments, S.B. and K.H. performed the experiments and analyzed the data, S.B. prepared the figures and performed the formal analysis, P.D.R. and S.C. helped with the MeCP2 experiments and provided the primary neurons, S.B. wrote the manuscript with input from O.R., L.S., S.C., K.H. and P.D.R.

\section{Data and material accessibility:}

Raw microscopy files, metadata, code and data tables are available on:

https://github.com/shaharbr . 


\section{Supplementary Materials}

\section{$\underline{\text { Not In Host Cell Nucleus }}$}
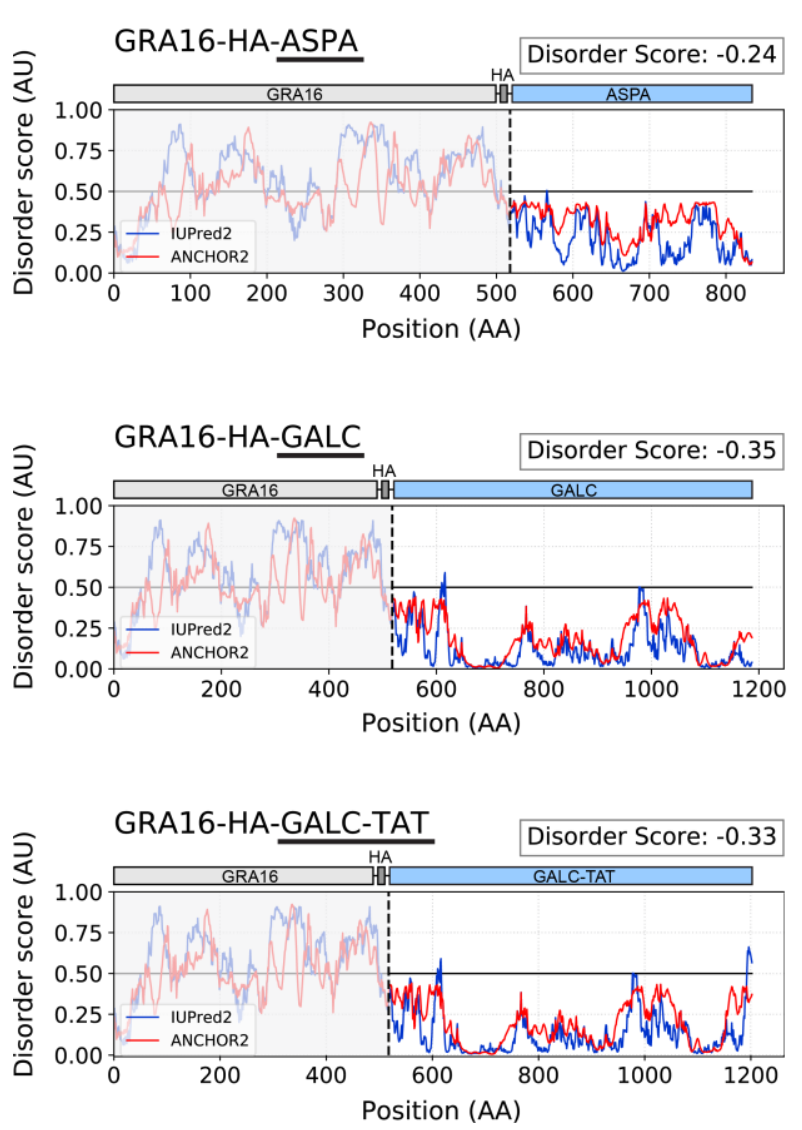

\section{$\underline{\text { In Host Cell Nucleus }}$}
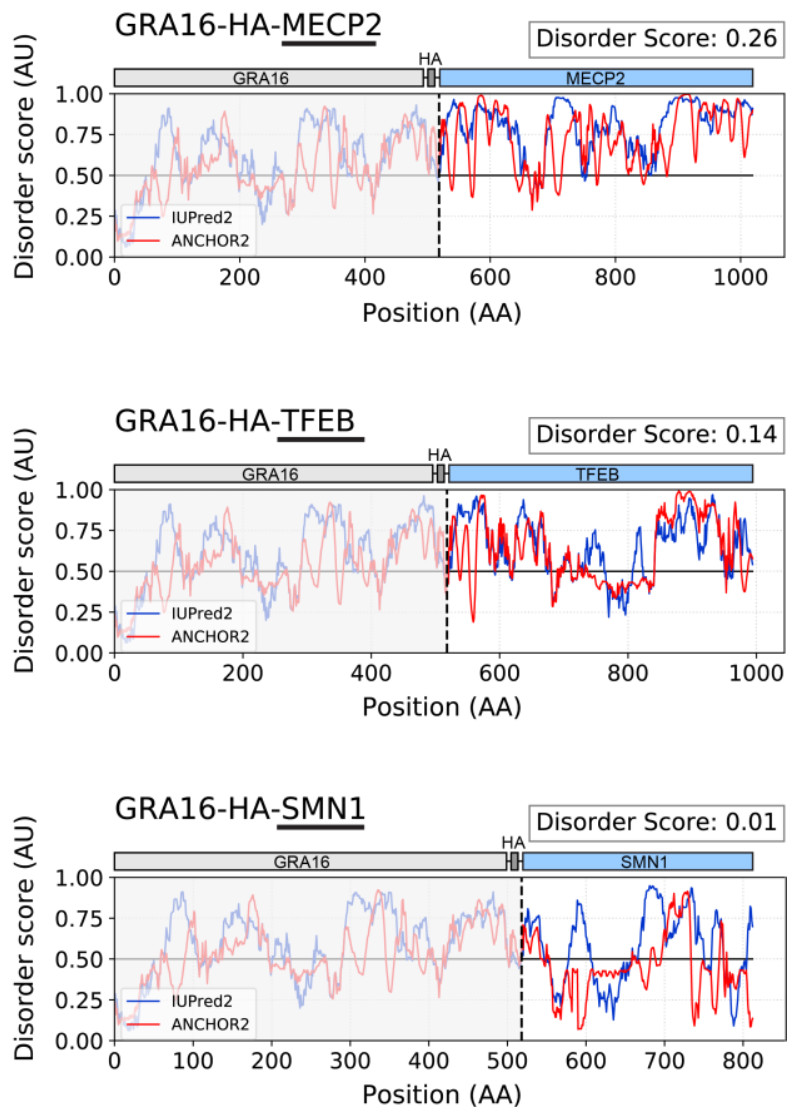

Fig. S1. The level of intrinsic disorder of the GRA16-fused therapeutic proteins predicts

\section{their localization to the host nucleus.}

435 Prediction of protein intrinsic disorder of GRA16 fusion proteins, using IUPred2 (disordered protein regions) and ANCHOR2 (disordered binding regions). Grey: GRA16-HA. Dashed lined marks the start of the heterologous protein. Disorder score = average(IUPred2 score, ANCHOR2 score) of the fused therapeutic protein. 

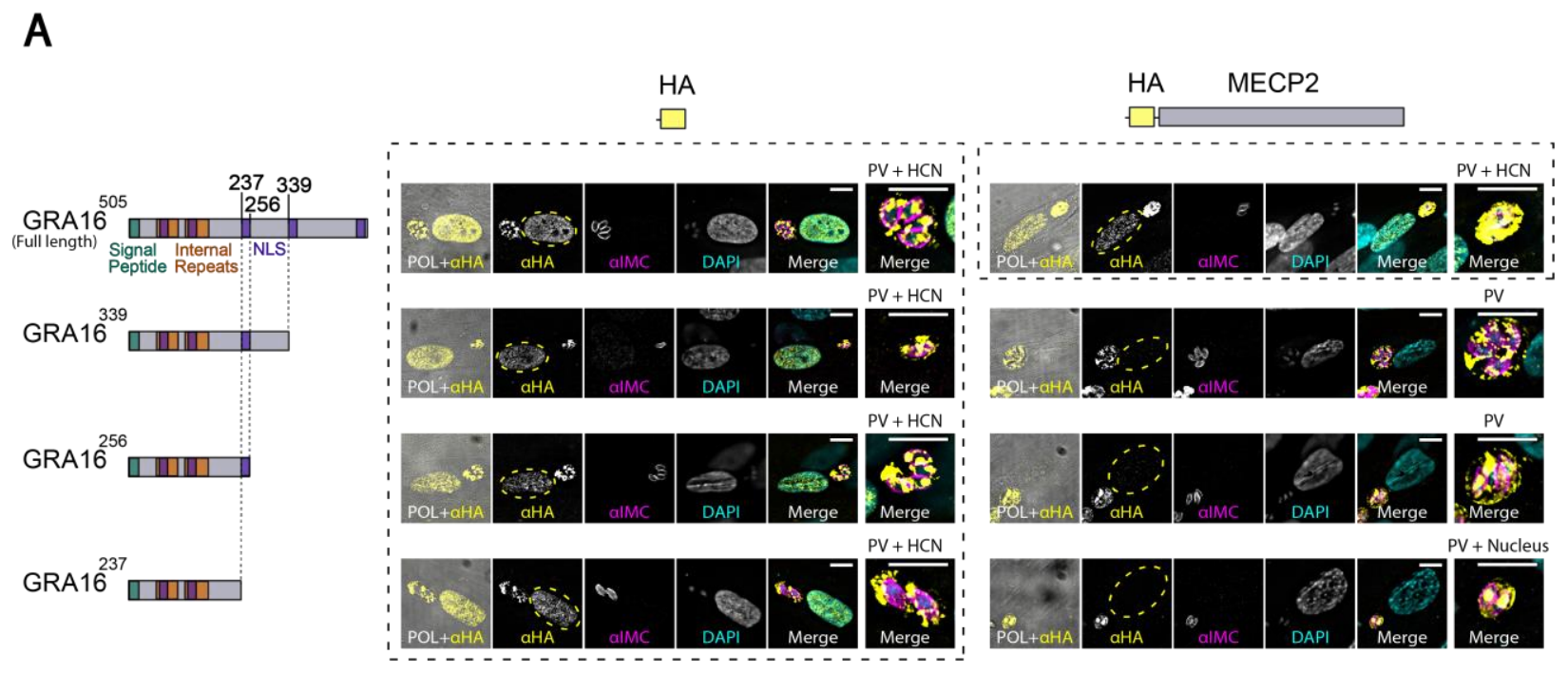

B
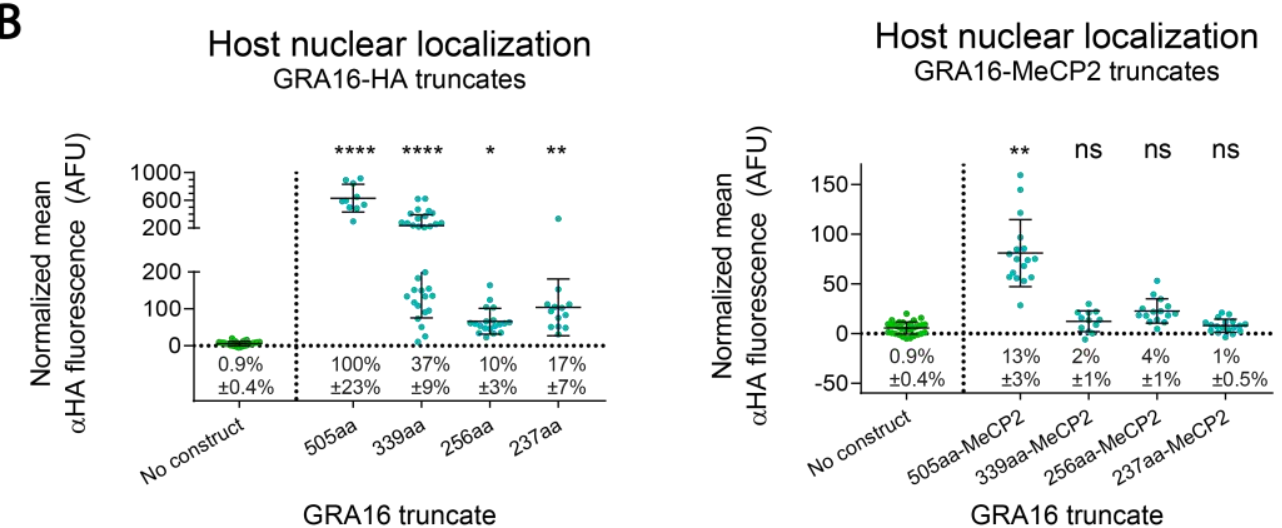

Fig. S2. Truncates of GRA16 do not deliver MeCP2 to the host nucleus

(A) Intracellular T. gondii stably expressing different fragments of GRA16, fused to either a HA tag alone or to an HA-tagged murine MeCP2 (codon optimized). All tagged proteins localized to the PV. However, only the GRA16 truncates without MeCP2 localized to the host cell nucleus

445 (HCN), as well as the full-length GRA16 fused to MeCP2. Rightmost image of each set shows a close-up of the parasite vacuole, and the text above it indicates the localization of the tagged protein in the image. Yellow dashed lines mark the host cell nucleus. Top and left: illustration of the full-length GRA16 (Bougdour et al., 2013)), C-terminally truncated GRA16, and the fused HA or HA-MECP2 sequences used in the respective constructs. NLS- Nuclear localization 
450 signal. (B) Fluorescence quantification of the anti-HA signal in the nucleus of infected host cells. Error bars represent mean $\pm \mathrm{SD}$. $\mathrm{N}=10$-32 cells per condition. Take note that the $\mathrm{y}$-axis of the leftmost graph is divided to two segments, to account for the high values recorded from GRA16HA. AFU- arbitrary fluorescence units. Text below each scatter plot shows the mean relative nuclear localization $\pm 95 \%$ confidence interval (relative to the full-length GRA16[505]-HA).

455 Significance represent the difference between the protein and the parental strain ('no construct'), calculated by two-way ANOVA with multiple comparisons (Dunnett test). **** $\mathrm{P}<0.0001 ; * *$ $\mathrm{P}<0.0021 ; * \mathrm{P}<0.0332$; ns- not significant. Scale bar: $10 \mu \mathrm{m}$. 


\begin{tabular}{|c|c|c|}
\hline Construct & Predicted size & Localization in $T$. gondii \\
\hline Toxofilin-HA-ASPA & $\begin{array}{l}64.4 \mathrm{kDa} \\
(27.1+1.5+35.7)\end{array}$ & Other (ER, microneme) \\
\hline Toxofilin-HA-ASPAopt & $\begin{array}{l}64.4 \mathrm{kDa} \\
(27.1+1.5+35.7)\end{array}$ & Other (ER, microneme) \\
\hline Toxofilin-HA-MECP2 & $\begin{array}{l}82.2 \mathrm{kDa} \\
(27.1+1.5+53.6)\end{array}$ & Other (ER, golgi, nucleus) \\
\hline Toxofilin-HA-MECP2opt & $\begin{array}{l}82.2 \mathrm{kDa} \\
(27.1+1.5+53.6)\end{array}$ & Other (ER, golgi, apicoplast, nucleus) \\
\hline Toxofilin-HA-GALC & $\begin{array}{l}103.8 \mathrm{kDa} \\
(27.1+1.5+75.1)\end{array}$ & Other (ER) \\
\hline Toxofilin-HA-GALCopt & $\begin{array}{l}105.7 \mathrm{kDa} \\
(27.1+1.5+77.1)\end{array}$ & Other (ER) \\
\hline Toxofilin-HA-GALC-TAT & $\begin{array}{l}105.6 \mathrm{kDa} \\
(27.1+1.5+76.9)\end{array}$ & Other (ER) \\
\hline Toxofilin-HA-SMN1 & $\begin{array}{l}60.5 \mathrm{kDa} \\
(27.1+1.5+31.9)\end{array}$ & Other (ER, golgi) \\
\hline Toxofilin-HA-GDNF & $\begin{array}{l}52.4 \mathrm{kDa} \\
(27.1+1.5+23.7)\end{array}$ & Rhoptries, Other (ER) \\
\hline Toxofilin-HA-PARK2 & $\begin{array}{l}71.1 \mathrm{kDa} \\
(27.1+1.5+42.4)\end{array}$ & Rhoptries, Other (ER) \\
\hline Toxofilin-HA-TFEBopt & $\begin{array}{l}\text { 81.5kDa } \\
(27.1+1.5+52.9)\end{array}$ & Rhoptries, Other (ER, microneme) \\
\hline
\end{tabular}

\section{Table S1. Toxofilin fusion constructs used in this study.}

Right column summarized the intracellular localizations in T. gondii observed for each construct.

Constructs that presented correct localizations to the targeted rhoptry secretion organelle are

475 highlighted in bold text. 


\begin{tabular}{|c|c|c|}
\hline Construct & Predicted size & Localization in $T$. gondii \\
\hline GRA16-HA-ASPA & $\begin{array}{l}92.2 \mathrm{kDa} \\
(54.9+1.5+35.7)\end{array}$ & PV, Other (ER, golgi, nucleus) \\
\hline GRA16-HA-ASPAopt & $\begin{array}{l}92.2 \mathrm{kDa} \\
(54.9+1.5+35.7)\end{array}$ & PV, Other (ER, golgi, nucleus) \\
\hline GRA16-HA-GALC & $\begin{array}{l}131.6 \mathrm{kDa} \\
(54.9+1.5+75.1)\end{array}$ & PV, Other (ER) \\
\hline GRA16-HA-GALCopt & $\begin{array}{l}133.5 \mathrm{kDa} \\
(54.9+1.5+77.1)\end{array}$ & Other $(\mathrm{ER})$ \\
\hline GRA16-HA-GALC-TAT & $\begin{array}{l}133.3 \mathrm{kDa} \\
(54.9+1.5+76.9)\end{array}$ & Not expressed \\
\hline GRA16-HA-MECP2opt & $\begin{array}{l}\text { 110kDa } \\
(54.9+1.5+53.6)\end{array}$ & PV + Host Cell Nucleus, Other (ER, golgi) \\
\hline GRA16-HA-SMN1 & $\begin{array}{l}\text { 88.3kDa } \\
(54.9+1.5+31.9)\end{array}$ & $\begin{array}{l}\text { PV + Host Cell Nucleus, Other (ER, golgi, } \\
\text { apicoplast, microneme) }\end{array}$ \\
\hline GRA16-HA-TFEBopt & $\begin{array}{l}\text { 109.3kDa } \\
(54.9+1.5+52.9)\end{array}$ & PV + Host Cell Nucleus, Other (ER) \\
\hline
\end{tabular}

Table S2. GRA16 fusion constructs used in this study.

Right column summarized the intracellular localizations in T. gondii observed for each construct.

Constructs that presented localizations to the PV and host cell nucleus are highlighted in bold text. 


\section{Materials and methods}

\section{T. gondii culture and maintenance}

Type I RH T. gondii were grown in human foreskin fibroblasts (HFF) in high glucose

Fetal Bovine Serum (FBS) and 1\% penicillin/streptomycin or $20 \mu \mathrm{g} / \mathrm{ml}$ gentamicin antibiotics, thereby referred to as "complete DMEM". Cultures were monitored daily, and the parasites were passaged by transferring a drop $(20-100 \mu \mathrm{l})$ of the supernatant of a lysed dish (containing extracellular parasites) into a fresh dish with confluent HFF cells.

\section{DNA transfection of $T$. gondii}

Tachyzoites of $T$. gondii were collected from the supernatant of lysed cells or mechanically released by scraping and passing through a 23-26 gauge needle. Extracellular parasites were filtered using a $3 \mu \mathrm{m}$-pore filter and pelleted by centrifugation at $800 \mathrm{G}$ for 5 minutes. Supernatant was discarded, and parasites were resuspended in cytomix buffer $(10 \mathrm{mM}$ K2HPO4/KH2PO4, 25 mM HEPES, 2 mM EGTA pH 7.6, 120 mM KCl, 0.15 mM CaCl2, 5 mM $\mathrm{MgCl} 2$ with $5 \mathrm{mM} \mathrm{KOH}$ adjusted to $\mathrm{pH} 7.6$ ) freshly supplemented with $3 \mathrm{mM}$ ATP and $3 \mathrm{mM}$ GSH. Resuspended parasites in a total volume of $350 \mu \mathrm{l}$ (for the BTX system) or $800 \mu \mathrm{l}$ (for the Bio-Rad system) were placed in an electroporation cuvette and supplemented with up to $50 \mu 1$ of 510 the transfected DNA (10-80 $\mu \mathrm{g}$ DNA). The DNA constructs were transfected into the parasites as either circular plasmids or after linearization with ScaI. The parasites were electroporated using two square-wave pulses. On the BTX ECM 830 square wave electroporator, we used the High Voltage (HV) mode and the following settings: voltage $1700 \mathrm{~V}$, pulse length 0.05 milliseconds, 2 
pulses, 200 milliseconds interval, unipolar. On the Bio-Rad GenePulser Xcell electroporator, we used the square wave protocol (program 3) with the following settings: voltage $1700 \mathrm{~V}$, pulse length 0.2 milliseconds, 2 pulses, 5 seconds interval, $4 \mathrm{~mm}$ cuvette. The transfected parasites were transferred onto cells seeded on glass coverslips. The cells were fixed 24 and 48 hours after infection, immunofluorescently stained and microscopically assessed for transfection efficiency, transient protein expression (expression from extrachromosomal DNA) and localization.

\section{Generation of $T$. gondii clonal lines}

To generate stable clonal lines, T. gondii were transfected as described, and genomic integration of the exogenous DNA was selected for by drug selection followed by clone isolation. After transfection, parasites were transferred onto a fresh dish with HFF cells. The next day, media was changed to fresh media containing the drug used for selection (pyrimethamine for DHFR-TS selection or mycophenolic acid+Xanthine for HXGPRT positive selection). Starting from the first detection of egressed extracellular parasites in the culture supernatant, 50$200 \mu \mathrm{l}$ drops of the supernatant were passed daily to a second dish of HFF cells in selective media. When the parasites in the second dish started egressing from the HFF, drops of the supernatant were passed daily to a third dish, and so on. After the 3rd-4th dish started lysing (about 3 weeks after transfection), the parasites were considered a "stable pool" containing parasites that integrated the exogenous DNA construct into their genome. The stable pool was immunofluorescently stained to assess the percentage of construct-expressing parasites in the stable pool as well as to evaluate protein localization upon genomic integration of the construct.

535 Clonal lines were isolated in 96-well plates with HFF by limiting dilutions or by FACS sorting single parasites into each well. After 5-10 days of growth, clones were selected as wells 
containing a single plaque, and tested for integration of the construct via PCR using genomic lysate as template. Genomic lysates were prepared in lysis buffer (10\% proteinase K in TE buffer) by incubation at $60{ }^{\circ} \mathrm{C}$ for 60 minutes followed by $95{ }^{\circ} \mathrm{C}$ for 10 minutes. PCR-positive

540 clones were verified by immunofluorescent staining. Protein localization was determined morphologically based on comparison to the polarized light image, and to co-staining with DAPI and anti-IMC1. Each fusion protein localization was validated over 2-5 independent transfections. At least 100 parasites were microscopically assessed by eye per transfection, and at least $20 \mathrm{z}$-stack images were acquired for each transfection, for transient and for stable

545 expression (Tables S1 and S2).

\section{Culture, neuronal differentiation and infection of LUHMES cells}

Culturing and differentiation of LUHMES cells were carried according to Shah et al. (Shah et al., 2016). Undifferentiated and differentiated LUHMES cells were grown in $75 \mathrm{~cm}^{2}$ in sterile distilled water). Undifferentiated LUHMES cells were split every 3 days and seeded at $10^{6}$ cells per $75 \mathrm{~cm}^{2}$ flask. They were maintained in a proliferation media consisting of advanced DMEM/F12 media supplemented with 1x N2 serum-free supplement, 2 mM L-glutamine and 40 ng/ml beta-FGF. LUHMES cell were differentiated into morphologically and biochemically 
normal proliferation media. The next day (day 0), differentiation was initiated by changing the media to the differentiation media. 2 days later (day 2), the cells were dissociated using trypsin and seeded in new flasks at a density of $6 * 10^{6}$ cells per flask, or on coated glass coverslips in 24well plates at $0.3 * 10^{6}$ cells per well, in fresh differentiation media. On day 6 of differentiation (at which point the cells are mature neurons, as confirmed by anti-NeuN staining), tachyzoites of the selected $T$. gondii line were resuspended in differentiation media and administered to the neurons.

\section{Culture and infection of $\mathrm{N} 2 \mathrm{~A}$ cells}

N2A (Neuro2A, mouse neuroblastoma) cells were cultured in high glucose DMEM supplemented with $10 \%$ FBS, $1 \%$ penicillin/streptomycin, $2 \mathrm{mM}$ L-glutamine and $0.1 \mathrm{mM}$ MEM non-essential amino acids solution. The day before infection, they were seeded on glass coverslips. The next day, tachyzoites of the selected $T$. gondii lines resuspended in the N2A media and administered to the $\mathrm{N} 2 \mathrm{~A}$ cells at $\mathrm{MOI}=1$. The cells were fixed 24 hours after infection, and immunofluorescently stained as described.

\section{Preparation and infection of mice primary neuronal cultures}

The cortex and hippocampi of WT P1 pups were dissociated enzymatically using papain and mechanically using gentle pipettation. Cells were counted and seeded in a 24 -well plate on poly-l-lysin coated glass coverslips at a density of 100,000 cells per well, in neurobasal media washed with PBS, fixed and immunofluorescently stained as described. 


\section{Immunofluorescent staining (Immunofluorescence assay, IFA)}

Cells were grown on $13 \mathrm{~mm}$ glass coverslips in a 24 -well plates. At the respective timepoint, cells were fixed in 4\% PFA for $20 \mathrm{~min}$ at room temperature (RT). They were permeabilized and blocked by incubation with $2 \%$ blocking solution ( $2 \%$ bovine serum albumin, 0.2\% Triton-X in PBS) for 20 minutes at RT. Blocking solution was removed, coverslips were covered with primary antibodies in blocking buffer and incubated for 1 hour at RT. Following 3 washes with PBS (5 minutes, shaking), they were covered with secondary antibodies in blocking buffer and incubated for 40 minutes at RT, protected from light. Coverslips were washed in PBS 3 times, dipped briefly in water, blotted on paper to remove excess water and mounted on slides with Fluoromount-G mounting media containing DAPI (Southern Biotech, 0100-20). Slides were allowed to dry overnight at RT prior to imaging. For long term storage, slides were kept at $4{ }^{\circ} \mathrm{C}$ 595 in the dark.

\section{Microscopy data acquisition and analysis}

Unless specified otherwise, all slides were imaged using a DeltaVision Core microscope (AppliedPrecision) using a 100x objective. Images were handled using the Fiji distribution of

600 ImageJ, imported using the OME bio-formats plugin (Linkert et al., 2010) and deconvolved using the Diffraction PSF 3D and Iterative Deconvolution 3D plugins (Dougherty, 2005). All adjustments of brightness and contrast were linear and applied to the entire image equally. Background fluorescence was determined by sampling an "empty" area of the image, and maximum display threshold for the image was set to allow optimal visualization of the cell 605 structures and protein localization (e.g. in the host cell nucleus). All raw images, imaging 
metadata, and ImageJ macros detailing the processing protocol are available on:

https://github.com/shaharbr.

\section{Automated high-content imaging of the kinetics of $\boldsymbol{T}$. gondii infection and protein secretion}

HFF cells were seeded in five 384-microwell plates corresponding to five fixation time points $(8,16,24,32,40$ hours $)$, at a dilution of 3,500 cells per well in $50 \mu$ complete DMEM and given 2 days to reach confluency (calculated 4,500 cells per well). Tachyzoites of the lines GRA16-HA, GRA16-MeCP2 and GRA16-TFEB were syringe-egressed, filtered and counted in a Neubauer chamber haemocytometer, and diluted to the appropriate concentration. Wells were infected with MOI (multiplicity of infection) of 3, 1 or $0.33(13,500,4,500$ or 1,500 parasites in $20 \mu \mathrm{l}$ per well, respectively), or received media only ("MOI 0"). At each timepoint, the respective plate was washed with PBS and fixed manually with 4\% PFA. Each condition (parasite line+MOI+timepoint) was repeated over 32-40 wells. Immunostaining was performed using a Beckman Coulter Biomek FXp liquid handling robot with a Thermo Multidrop Reagent Dispenser and with a MANTIS Liquid Handler. Cells were first permeabilized and blocked with $2 \%$ blocking solution for $20 \mathrm{~min}$ at RT. Blocking buffer was removed and cells were incubated with $5 \mu \mathrm{l}$ per well of the primary antibody solution (anti-HA + anti-IMC1) in blocking solution, for 60 min at RT. Cells were washed 3 times with PBS, and then incubated with $20 \mu$ secondary antibody solution and Hoechst 33342 dye (diluted 1:50,000) in blocking solution, for 40 min at RT and protected from light. Cells were washed 3 times with PBS. Plates were then imaged on the GE IN Cell 2000 platform. 5 fields-of-view were acquired from each well (average 360 cells per field-of-view). 
LUHMES cells were differentiated to neurons in 24-well plates with coated glass coverslips using the protocol described above. On day 6 of differentiation, tachyzoites of the line GRA16-HA were administered at MOI 0.5, 1 or 2. On each timepoint $(6,1224$ and 32 hours post-infection), the respective wells were washed with PBS, fixed and immunofluorescently stained with anti-HA and anti-IMC1 or anti-HA and anti-NeuN manually as described. Each condition (MOI+timepoint) was repeated over 3 coverslips. 10-60 random regions-of-interest (average 80 neurons per image) were imaged for each coverslip on the BX63 Olympus microscope using a 40x objective.

\section{Analysis of T. gondii infection and protein delivery kinetics in HFF and in LUHMES neurons}

Image analysis was performed using the open-source CellProfiler (Carpenter et al., 2006) software. Full protocol used for the image analysis, including the parameters chosen for identification of host cells and intracellular T. gondii, is available together with the raw data and image analysis outputs on: https://github.com/shaharbr. In brief, host cell nuclei were identified using the DAPI channel and T. gondii were identified using the 594nm channel (corresponding to anti-IMC1 staining). Each T. gondii vacuole was associated to a host cell nucleus based on proximity. Fluorescence intensity on the $488 \mathrm{~nm}$ channel, corresponding to the anti-HA staining was used to quantify the levels of GRA16 fusion-protein localization in the parasite vacuoles and in the host cell nuclei. Importantly, timepoints above 24 hours post infection were removed from 
the dataset as we found that the parasite vacuoles were too large for efficient segmentation and host cell association by the used image analysis tool. Resulting data tables were analyzed using a custom python code. In brief, this analysis involved labeling and organization of the data, removal of images in which parasite identification failed, removal of outlier wells with exceptionally high fluorescence intensity ( $>5$ std from mean of condition), normalization by subtraction of background fluorescence, labeling of cells infected with a single parasite vacuole, labeling of nuclei positive for the tagged protein (threshold set as above $99 \%$ of uninfected cells), calculating descriptive statistics for each well $(\mathrm{N}=$ number of cells $)$ and for each condition $(\mathrm{N}=$ number of wells) and plotting. Full code and analysis outputs are available on:

https://github.com/shaharbr .

\section{Measuring host nuclear fluorescence intensity in HFF and neurons}

Cells infected with $T$. gondii expressing the respective construct were fixed at the

665 designated time points (24 hours for HFF, 12-24 hours for LUHMES), and immunofluorescently stained as described. Infected HFF were stained with anti-HA and anti-IMC1, and infected LUHMES neurons were stained with anti-HA and anti-MeCP2 (for quantifying nuclear MeCP2 in infected neurons) or with anti-NeuN and anti-MeCP2 (for verifying differentiation), and imaged. Mean nuclear intensity of the respective fluorescent signal was measured using imageJ's 'Measure' function.

For HFF, each infected cell nucleus was measured alongside 3 random background regions in the same image. To calculate the normalized mean nuclear intensity, the average of the three background regions was subtracted from the value recorded from the infected cell nucleus, and divided by the exposure time (to account for different exposure times used in 
675 imaging of the different transfections). Relative nuclear localization of the expressed protein was calculated by dividing each normalized intensity measurement by the mean normalized intensity of GRA16-HA.

For LUHMES neurons, all nuclei detected in each image were measured. For each measured nucleus, it was labeled whether the neuron contained a $T$. gondii vacuole, and whether 680 the nucleus was also HA-positive. Neurons that contained a T. gondii vacuole and were HApositive were considered 'infected' and neurons that did not contain a $T$. gondii vacuole and did not present nuclear anti-HA staining were considered 'uninfected'. Relative nuclear localization of MeCP2 was calculated by dividing each normalized intensity measurement by the mean normalized intensity of WT neurons (endogenously expressing hMeCP2) from the same 685 timepoint.

\section{Intrinsic disorder score and correlation to nuclear localization of the fusion proteins}

The intrinsic disorder profile for the full translated open read frames of each GRA16 fusion protein was calculated using https://iupred2a.elte.hu/, using the following parameters:

690 Prediction type: IUPred2 long disorder (default), Context-dependent prediction: ANCHOR2 (Mészáros et al., 2018). A custom python code was used to calculate the averaged intrinsic disorder score of the fused therapeutic protein, calculate the correlation between the intrinsic disorder score and nuclear localization of each fusion protein and generate plots. The averaged intrinsic disorder score was calculated as the average of the ANCHOR2 score and the IUPred2 score along the span of the fused therapeutic protein (excluding GRA16-HA), minus the 0.5 threshold for disorder. Protein disorder data and code are available on: https://github.com/shaharbr . 


\section{Molecular cloning}

For the plasmids encoding for Toxofilin-fused proteins, we used as a backbone a pGRA vector containing Toxofilin cDNA fused to Cre recombinase, surrounded by the $1.1 \mathrm{~kb}$ genomic sequence upstream to Toxofilin ('Toxofilin promoter') and the 3' UTR of GRA2, a kind gift from A. A. Koshy (Koshy et al., 2010; Lodoen et al., 2010). To generate the therapeutic fusion constructs, we used the following mammalian cDNA sequences: human SMN1 (Addgene, \#37057), human ASPA (DNASU, HsCD00044152), ASPAopt (GenScript, custom synthesis, human ASPA codon optimized for T. gondii), human PARK2 (MGC, BC022014), human GDNF (MGC, BC069119), murine MECP2 isoform 1e/B (GenScript, OMu23690), MECP2opt (GenScript, custom synthesis, murine MeCP2 isoform 1e/B codon optimized for T. gondii), TFEBopt (GenScript, custom synthesis, human TFEB isoform 1 codon optimized for T. gondii), human GALC (MGC, BC036518), GALCopt (GenScript, custom synthesis, human GALC isoform 1 [NP_000144] codon optimized for T. gondii).

To generate plasmids for the expression of Toxofilin-fused GDNF, PARK2 and MECP2, we PCR-amplified the mammalian cDNA with primers that add an EcoRV restriction site at the 5' and a PacI restriction site at the 3' of the cDNA. The Cre recombinase was replaced with the

715 amplified mammalian cDNA by restriction-ligation (using SfoI and PacI for the backbone and EcoRV and PacI for the cDNA, SfoI and EcoRV both produce blunt ends).

To generate plasmids for the expression of Toxofilin-fused ASPA, SMN1, GALC, GALCTAT and MECP2opt, we amplified the pGRA vector and cDNA, and used NEBuilder assembly. As GALC contains a region of exceptionally high $\% \mathrm{GC}$ at the start of the gene, PCR amplification and NEBuilder assembly resulted in a deletion at the start of the gene. To fix this, 
we performed an additional cloning step to add the missing fragment using the 'round-the-horn'

(Stephen Floor, 2018) PCR blunt cloning method for both the Toxofilin-GALC and Toxofilin-

GALC-TAT plasmids.

To generate plasmids for the expression of Toxofilin-fused TFEBopt, ASPAopt and

GALCopt, we replaced the MEP2opt in the Toxofilin-MECP2opt plasmid with the respective cDNA using restriction-ligation with EcoRV and PacI which were added to the sequence during DNA synthesis.

To generate plasmids for the expression of GRA16-fused genes, GRA16 and its promoter were amplified from RH T. gondii genomic DNA (primers were designed based on (Bougdour et sequence in the Toxofilin-ASPA plasmid, with the GRA16 promoter and coding sequence, to produce the GRA16-ASPA plasmid (by NEBuilder assembly).

To generate plasmids for the expression of GRA16-fused MECP2opt, SMN1, GALC, GALC-TAT, TFEBopt, GALCopt and ASPAopt, we replaced the ASPA with the respective cDNA using restriction-ligation with EcoRV+PacI.

GRA16 truncate vectors ( $H A$-fused and $H A-M E C P 2$-fused) were generate from the GRA16-HA and GRA16-MECP2opt vectors using the 'round-the-horn' method.

\section{Antibodies}

Antibodies and the respective concentrations they were used in, are as follows: anti-HA

(Sigma-Aldrich ROAHAHA/Roche clone 3F10, 1:1000), anti-IMC1 (gift from Prof. Dominique Soldati-Favre, 1:2000), anti-MeCP2 (Cell Signaling \#3456, 1:200), anti-NeuN (Abcam ab104224, 1:500), Alexa Fluor Goat anti-Rat 488 and 594 (Invitrogen \#A-11006 and \#A-11007, 
1:1000), Alexa Fluor Goat anti-Rabbit 488 and 594 (Invitrogen \#A-11008, \#A-11012, 1:1000),

745 Alexa Fluor anti-mouse 488 and 594 (Invitrogen \#A-11001 and \#A-11005, 1:1000).

\section{Statistical analysis}

To determine the significance of nuclear localization of each GRA16-fused protein, we performed one-way ANOVA for the effect of the expressed construct, with multiple comparisons to the "no construct" control. To determine the significance of nuclear localization of each truncated variant of GRA16, fused to HA or to HA-MeCP2, we performed two-way ANOVA for the effect of the GRA16 variant (rows) and of the fused sequence (HA or HA-MECP2, columns), with multiple comparisons to the "no construct" control. To determine the significance of the difference in infection rate between parasite lines in the kinetics analysis, for each timepoint we performed two-way ANOVA for the effect of parasite line (columns) and MOI (rows). To determine the significance of nuclear levels of MeCP2 and GRA16-MeCP2, we performed twoway ANOVA for the effect of the time after inoculation (rows) and the condition (columns), with multiple comparisons to the to the "uninfected MECP2-KO" control. Multiple comparisons were performed using the following parameters: within each row, compare the means of each group to control, report multiplicity adjusted $\mathrm{P}$ value for each comparison (Dunnett test), one family per row.

\section{References:}

765 Allen, S. J., Watson, J. J., Shoemark, D. K., Barua, N. U., \& Patel, N. K. (2013). GDNF, NGF and BDNF as therapeutic options for neurodegeneration. Pharmacology \& Therapeutics, 138(2), 155-175. https://doi.org/10.1016/j.pharmthera.2013.01.004 
Berenreiterová, M., Flegr, J., Kuběna, A. A., \& Němec, P. (2011). The distribution of Toxoplasma gondii cysts in the brain of a mouse with latent toxoplasmosis: implications for the behavioral manipulation hypothesis. PloS One, 6(12), e28925. https://doi.org/10.1371/journal.pone.0028925

Boothroyd, J. C., \& Dubremetz, J.-F. (2008). Kiss and spit: the dual roles of Toxoplasma rhoptries. Nature Reviews. Microbiology, 6(1), 79-88. https://doi.org/10.1038/nrmicro1800

Bougdour, A., Durandau, E., Brenier-Pinchart, M.-P., Ortet, P., Barakat, M., Kieffer, S., ... Hakimi, M.-A. (2013). Host cell subversion by Toxoplasma GRA16, an exported dense granule protein that targets the host cell nucleus and alters gene expression. Cell Host \& Microbe, 13(4), 489-500. https://doi.org/10.1016/j.chom.2013.03.002

Bowerman, M., Becker, C. G., Yáñez-Muñoz, R. J., Ning, K., Wood, M. J. A., Gillingwater, T. H., ... UK SMA Research Consortium, T. U. S. R. (2017). Therapeutic strategies for spinal muscular atrophy: SMN and beyond. Disease Models \& Mechanisms, 10(8), 943-954. https://doi.org/10.1242/dmm.030148

Cabral, C. M., Tuladhar, S., Dietrich, H. K., Nguyen, E., MacDonald, W. R., Trivedi, T., ... Koshy, A. A. (2016). Neurons are the Primary Target Cell for the Brain-Tropic Intracellular Parasite Toxoplasma gondii. PLoS Pathogens, 12(2), e1005447. https://doi.org/10.1371/journal.ppat.1005447

Carpenter, A. E., Jones, T. R., Lamprecht, M. R., Clarke, C., Kang, I., Friman, O., ... Sabatini, D. M. (2006). CellProfiler: image analysis software for identifying and quantifying cell phenotypes. Genome Biology, 7(10), R100. https://doi.org/10.1186/gb-2006-7-10-r100

Carrette, L. L. G., Blum, R., Ma, W., Kelleher, R. J., \& Lee, J. T. (2018). Tsix-Mecp2 female mouse model for Rett syndrome reveals that low-level MECP2 expression extends life and improves neuromotor function. Proceedings of the National Academy of Sciences of the United States of America, 201800931. https://doi.org/10.1073/pnas.1800931115

Colella, P., Ronzitti, G., \& Mingozzi, F. (2018). Emerging Issues in AAV-Mediated In Vivo Gene Therapy. Molecular Therapy - Methods \& Clinical Development, 8, 87-104. https://doi.org/10.1016/J.OMTM.2017.11.007

Collins, A. L., Levenson, J. M., Vilaythong, A. P., Richman, R., Armstrong, D. L., Noebels, J. L., ... Zoghbi, H. Y. (2004). Mild overexpression of MeCP2 causes a progressive neurological disorder in mice. Human Molecular Genetics, 13(21), 2679-2689. 
https://doi.org/10.1093/hmg/ddh282

800 Curt-Varesano, A., Braun, L., Ranquet, C., Hakimi, M.-A., \& Bougdour, A. (2016). The aspartyl protease TgASP5 mediates the export of the Toxoplasma GRA16 and GRA24 effectors into host cells. Cellular Microbiology, 18(2), 151-167. https://doi.org/10.1111/cmi.12498

DiNunzio, J. C., \& Williams, R. O. (2008). CNS Disorders-Current Treatment Options and the Prospects for Advanced Therapies. Drug Development and Industrial Pharmacy, 34(11), 1141-1167. https://doi.org/10.1080/03639040802020536

Dougherty, R. (2005). Extensions of DAMAS and Benefits and Limitations of Deconvolution in Beamforming. In 11th AIAA/CEAS Aeroacoustics Conference. Reston, Virigina: American Institute of Aeronautics and Astronautics. https://doi.org/10.2514/6.2005-2961

Fuge, O., Vasdev, N., Allchorne, P., \& Green, J. S. (2015). Immunotherapy for bladder cancer. Research and Reports in Urology, 7, 65-79. https://doi.org/10.2147/RRU.S63447

Gadalla, K. K. E., Bailey, M. E. S., Spike, R. C., Ross, P. D., Woodard, K. T., Kalburgi, S. N., ... Cobb, S. R. (2013). Improved survival and reduced phenotypic severity following AAV9/MECP2 gene transfer to neonatal and juvenile male Mecp2 knockout mice. Molecular Therapy: The Journal of the American Society of Gene Therapy, 21(1), 18-30. https://doi.org/10.1038/mt.2012.200

Gadalla, K. K. E., Vudhironarit, T., Hector, R. D., Sinnett, S., Bahey, N. G., Bailey, M. E. S., ... Cobb, S. R. (2017). Development of a Novel AAV Gene Therapy Cassette with Improved Safety Features and Efficacy in a Mouse Model of Rett Syndrome. Molecular Therapy Methods \& Clinical Development, 5, 180-190. https://doi.org/10.1016/j.omtm.2017.04.007 methods for genome engineering. Trends in Biotechnology, 31(7), 397-405. https://doi.org/10.1016/J.TIBTECH.2013.04.004

Garg, S. K., Lioy, D. T., Cheval, H., McGann, J. C., Bissonnette, J. M., Murtha, M. J., ... Mandel, G. (2013). Systemic delivery of MeCP2 rescues behavioral and cellular deficits in female mouse models of Rett syndrome. The Journal of Neuroscience : The Official Journal of the Society for Neuroscience, 33(34), 13612-13620. https://doi.org/10.1523/JNEUROSCI.1854-13.2013

Guy, J., Alexander-Howden, B., FitzPatrick, L., DeSousa, D., Koerner, M. V, Selfridge, J., \& Bird, A. (2018). A mutation-led search for novel functional domains in MeCP2. Human 
Molecular Genetics, 27(14), 2531-2545. https://doi.org/10.1093/hmg/ddy159

Guy, J., Gan, J., Selfridge, J., Cobb, S., \& Bird, A. (2007). Reversal of neurological defects in a mouse model of Rett syndrome. Science (New York, N.Y.), 315(5815), 1143-1147. https://doi.org/10.1126/science.1138389

Hakimi, M.-A., \& Bougdour, A. (2015). Toxoplasma's ways of manipulating the host transcriptome via secreted effectors. Current Opinion in Microbiology, 26, 24-31. https://doi.org/10.1016/J.MIB.2015.04.003

Hakimi, M.-A., Olias, P., \& Sibley, L. D. (2017). ToxoplasmaEffectors Targeting Host Signaling and Transcription. Clinical Microbiology Reviews, 30(3), 615-645. https://doi.org/10.1128/CMR.00005-17

840 Helmby, H. (2015). Human helminth therapy to treat inflammatory disorders - where do we stand? BMC Immunology, 16, 12. https://doi.org/10.1186/s12865-015-0074-3

Hill, D. E., Chirukandoth, S., \& Dubey, J. P. (2005). Biology and epidemiology of Toxoplasma gondii in man and animals. Animal Health Research Reviews, 6(1), 41-61. Retrieved from http://www.ncbi.nlm.nih.gov/pubmed/16164008

Jimenez-Ruiz, E., Wong, E. H., Pall, G. S., \& Meissner, M. (2014). Advantages and disadvantages of conditional systems for characterization of essential genes in Toxoplasma gondii. Parasitology, 141(11), 1390-1398. https://doi.org/10.1017/S0031182014000559

Karra, D., \& Dahm, R. (2010). Transfection techniques for neuronal cells. The Journal of Neuroscience : The Official Journal of the Society for Neuroscience, 30(18), 6171-6177. https://doi.org/10.1523/JNEUROSCI.0183-10.2010

Katz, D. M., Bird, A., Coenraads, M., Gray, S. J., Menon, D. U., Philpot, B. D., \& Tarquinio, D. C. (2016). Rett Syndrome: Crossing the Threshold to Clinical Translation. Trends in Neurosciences, 39(2), 100-113. https://doi.org/10.1016/j.tins.2015.12.008

Koshy, A. A., Dietrich, H. K., Christian, D. A., Melehani, J. H., Shastri, A. J., Hunter, C. A., \& Boothroyd, J. C. (2012). Toxoplasma co-opts host cells it does not invade. PLoS Pathogens, 8(7), e1002825. https://doi.org/10.1371/journal.ppat.1002825

Koshy, A. A., Fouts, A. E., Lodoen, M. B., Alkan, O., Blau, H. M., \& Boothroyd, J. C. (2010). Toxoplasma secreting Cre recombinase for analysis of host-parasite interactions. Nature Methods, 7(4), 307-309. https://doi.org/10.1038/nmeth.1438 
pharmacological classification. Nature Reviews Drug Discovery, 7(1), 21-39.

https://doi.org/10.1038/nrd2399

Leone, P., Shera, D., McPhee, S. W. J., Francis, J. S., Kolodny, E. H., Bilaniuk, L. T., ... Janson, C. G. (2012). Long-Term Follow-Up After Gene Therapy for Canavan Disease. Science Translational Medicine, 4(165), 165ra163-165ra163.

https://doi.org/10.1126/scitranslmed.3003454

Linkert, M., Rueden, C. T., Allan, C., Burel, J.-M., Moore, W., Patterson, A., ... Swedlow, J. R. (2010). Metadata matters: access to image data in the real world. The Journal of Cell Biology, 189(5), 777-782. https://doi.org/10.1083/jcb.201004104

Lodoen, M. B., Gerke, C., \& Boothroyd, J. C. (2010). A highly sensitive FRET-based approach reveals secretion of the actin-binding protein toxofilin during Toxoplasma gondii infection. Cellular Microbiology, 12(1), 55-66. https://doi.org/10.1111/j.1462-5822.2009.01378.x

Lotharius, J., Barg, S., Wiekop, P., Lundberg, C., Raymon, H. K., \& Brundin, P. (2002). Effect of mutant alpha-synuclein on dopamine homeostasis in a new human mesencephalic cell line. The Journal of Biological Chemistry, 277(41), 38884-38894. https://doi.org/10.1074/jbc.M205518200

Lu, T. K., Mimee, M., Citorik, R. J., \& Pepper, K. (2017). Engineering the Microbiome for Human Health Applications. In The Chemistry of Microbiomes: Proceedings of a Seminar Series. Washington, D.C.: National Academies Press. https://doi.org/10.17226/24751 postmitotic neurons rescues Rett syndrome in mice. Proceedings of the National Academy of Sciences of the United States of America, 101(16), 6033-6038. https://doi.org/10.1073/pnas.0401626101

Lyst, M. J., \& Bird, A. (2015). Rett syndrome: a complex disorder with simple roots. Nature Reviews. Genetics, 16(5), 261-275. https://doi.org/10.1038/nrg3897

Marino, N. D., Panas, M. W., Franco, M., Theisen, T. C., Naor, A., Rastogi, S., .. Boothroyd, J. C. (2018). Identification of a novel protein complex essential for effector translocation across the parasitophorous vacuole membrane of Toxoplasma gondii. PLOS Pathogens, 14(1), e1006828. https://doi.org/10.1371/journal.ppat.1006828

890 Matagne, V., Ehinger, Y., Saidi, L., Borges-Correia, A., Barkats, M., Bartoli, M., ... Roux, J.-C. (2017). A codon-optimized Mecp2 transgene corrects breathing deficits and improves 
survival in a mouse model of Rett syndrome. Neurobiology of Disease, 99, 1-11. https://doi.org/10.1016/J.NBD.2016.12.009

Mendez, O. A., \& Koshy, A. A. (2017). Toxoplasma gondii: Entry, association, and physiological influence on the central nervous system. PLOS Pathogens, 13(7), e1006351. https://doi.org/10.1371/journal.ppat.1006351

Meng, X.-L., Eto, Y., Schiffmann, R., \& Shen, J.-S. (2013). HIV Tat Domain Improves Crosscorrection of Human Galactocerebrosidase in a Gene- and Flanking Sequence-dependent Manner. Molecular Therapy. Nucleic Acids, 2, e130. https://doi.org/10.1038/mtna.2013.57 protein disorder as a function of redox state and protein binding. Nucleic Acids Research, 46(W1), W329-W337. https://doi.org/10.1093/nar/gky384

Mochizuki, H. (2007). Gene therapy for Parkinson's disease. Expert Review of Neurotherapeutics, 7(8), 957-960. https://doi.org/10.1586/14737175.7.8.957

Napolitano, G., \& Ballabio, A. (2016). TFEB at a glance. Journal of Cell Science, 129(13), 2475-2481. https://doi.org/10.1242/jcs.146365

Pittman, K. J., \& Knoll, L. J. (2015). Long-Term Relationships: the Complicated Interplay between the Host and the Developmental Stages of Toxoplasma gondii during Acute and Chronic Infections. Microbiology and Molecular Biology Reviews, 79(4), 387-401. https://doi.org/10.1128/MMBR.00027-15

Rafi, M. A., Rao, H. Z., Luzi, P., Curtis, M. T., \& Wenger, D. A. (2012). Extended normal life after AAVrh10-mediated gene therapy in the mouse model of Krabbe disease. Molecular Therapy: The Journal of the American Society of Gene Therapy, 20(11), 2031-2042. https://doi.org/10.1038/mt.2012.153

915 Ramocki, M. B., Tavyev, Y. J., \& Peters, S. U. (2010). The MECP2 duplication syndrome. American Journal of Medical Genetics. Part A, 152A(5), 1079-1088. https://doi.org/10.1002/ajmg.a.33184

Ross, P. D., Guy, J., Selfridge, J., Kamal, B., Bahey, N., Tanner, K. E., ... Cobb, S. (2016). Exclusive expression of $\mathrm{MeCP} 2$ in the nervous system distinguishes between brain and peripheral Rett syndrome-like phenotypes. Human Molecular Genetics, 25(20), ddw269. https://doi.org/10.1093/hmg/ddw269

Scholz, D., Pöltl, D., Genewsky, A., Weng, M., Waldmann, T., Schildknecht, S., \& Leist, M. 
(2011). Rapid, complete and large-scale generation of post-mitotic neurons from the human LUHMES cell line. Journal of Neurochemistry, 119(5), 957-971.

Shah, R. R., Cholewa-Waclaw, J., Davies, F. C. J., Paton, K. M., Chaligne, R., Heard, E., ... Bird, A. P. (2016). Efficient and versatile CRISPR engineering of human neurons in culture to model neurological disorders. Wellcome Open Research, 1, 13. https://doi.org/10.12688/wellcomeopenres.10011.1

Sinnett, S. E., Hector, R. D., Gadalla, K. K. E., Heindel, C., Chen, D., Zaric, V., ... Gray, S. J. (2017). Improved MECP2 Gene Therapy Extends the Survival of MeCP2-Null Mice without Apparent Toxicity after Intracisternal Delivery. Molecular Therapy - Methods \& Clinical Development, 5, 106-115. https://doi.org/10.1016/J.OMTM.2017.04.006

Stephen Floor. (2018). Around-the-horn PCR and cloning. https://doi.org/dx.doi.org/10.17504/protocols.io.rf2d3qe

Torchilin, V. (2008). Intracellular delivery of protein and peptide therapeutics. Drug Discovery Today: Technologies, 5(2-3), e95-e103. https://doi.org/10.1016/J.DDTEC.2009.01.002 Wang, J.-L., Huang, S.-Y., Behnke, M. S., Chen, K., Shen, B., \& Zhu, X.-Q. (2016). The Past, Present, and Future of Genetic Manipulation in Toxoplasma gondii. Trends in Parasitology, 32(7), 542-553. https://doi.org/10.1016/J.PT.2016.04.013

Whitrow, M. (1990). Wagner-Jauregg and fever therapy. Medical History, 34(3), 294-310. Retrieved from http://www.ncbi.nlm.nih.gov/pubmed/2214949

Wood, L. M., \& Paterson, Y. (2014). Attenuated Listeria monocytogenes: a powerful and versatile vector for the future of tumor immunotherapy. Frontiers in Cellular and Infection Microbiology, 4, 51. https://doi.org/10.3389/fcimb.2014.00051 